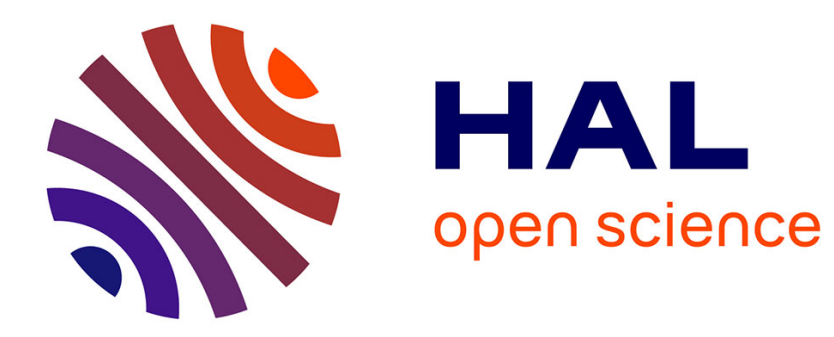

\title{
Emploi de lasers dans la technologie des photopiles
} J.C. Muller, P. Siffert

\section{To cite this version:}

J.C. Muller, P. Siffert. Emploi de lasers dans la technologie des photopiles. Revue de Physique Appliquée, 1980, 15 (3), pp.611-629. 10.1051/rphysap:01980001503061100 . jpa-00244768

\section{HAL Id: jpa-00244768 https://hal.science/jpa-00244768}

Submitted on 1 Jan 1980

HAL is a multi-disciplinary open access archive for the deposit and dissemination of scientific research documents, whether they are published or not. The documents may come from teaching and research institutions in France or abroad, or from public or private research centers.
L'archive ouverte pluridisciplinaire HAL, est destinée au dépôt et à la diffusion de documents scientifiques de niveau recherche, publiés ou non, émanant des établissements d'enseignement et de recherche français ou étrangers, des laboratoires publics ou privés. 


\title{
Emploi de lasers dans la technologie des photopiles
}

\author{
J. C. Muller et P. Siffert \\ Centre de Recherches Nucléaires, Groupe de Physique et Applications des Semiconducteurs (Phase), \\ 67037 Strasbourg Cedex, France
}

(Reçu le 22 septembre 1979, révisé le 28 novembre 1979, accepté le 3 décembre 1979)

\begin{abstract}
Résumé. - Dans cet article on passe en revue les différentes applications des lasers de puissance continus ou impulsionnels dans la technologie des cellules solaires à base de silicium. On considère tout d'abord les différents modèles développés pour calculer les effets de ces flux lumineux intenses sur le silicium; puis, on analyse les possibilités d'emploi de ces dispositifs pour préparer des films autosupportants ou des couches sur substrat. Finalement, trois méthodes de réalisation de la jonction sont décrites : activation de dopants diffusés, implantation ionique conventionnelle ou simplifiée et dépôt de films en surface.
\end{abstract}

\begin{abstract}
In this paper, the various possibilities existing for the use of high power density continuous or pulsed lasers in the technology of silicon solar cells are reviewed. First, the models developped for calculating the effects of the absorption of the high intensity light on silicon are considered. In a second part, the methods developped for manufacturing either self supporting films or deposited layers are described. Finally, three methods for preparing the rectifying junction are considered : activation of diffused dopants, conventional as well as simplified ion implantation, alloying films deposited on surface.
\end{abstract}

Introduction. - Presque simultanément, plusieurs groupes russes [1-11] ont montré en 1976 qu'il est possible de recristalliser une couche superficielle de silicium endommagée par bombardement ionique au moyen d'une impulsion brève ou par balayage d'un faisceau focalisé issu d'un laser de puissance, essentiellement à rubis ou YAG. Ce résultat est tout d'abord passé inaperçu aux U.S.A., ce qui permit à quelques groupes européens d'entreprendre très tôt des travaux dans ce domaine, avant que ce sujet ne devienne un thème majeur de nombreux laboratoires à travers le monde. En 1978 fut organisée une première table ronde à Catania [12] et depuis lors plusieurs conférences sont consacrées entièrement ou partiellement à ce thème [13-18]. En effet, très vite on s'aperçut qu'en dehors de la recristallisation de couches implantées, il était possible de réaliser des couches épitaxiales sans recours à l'ultra-vide, des films supersaturés de dopants ainsi que des jonctions alliées.

Nous nous limiterons dans cet article de synthèse à l'application de cette technologie à l'élaboration des matériaux pour photopiles ou à la préparation des jonctions créant la barrière de potentiel. De plus, cet exposé sera limité au silicium, bien que quelques résultats aient été obtenus sur des cellules GaAs [19-21]

1. Effets d'un faisceau laser intense sur le silicium. 1.1 INTERACTION LASER-SILICIUM. - L'absorption d'un faisceau laser dans un semiconducteur obéit à des mécanismes intermédiaires entre ceux régissant l'absorption dans un diélectrique et dans un métal. Dans le premier cas, pour une forte excitation, l'absorption se fait directement par interaction avec le réseau. L'interaction de type métallique intervient lorsqu'il existe une densité importante de porteurs libres, qui absorbent l'énergie lumineuse pour la communiquer au réseau sous forme de chaleur. $\mathrm{Si}$ l'énergie de la radiation lumineuse est supérieure à celle d'activation du semiconducteur, des paires électron-trou sont générées près de la surface, qui vont se recombiner en transitions non radiatives et finalement fournir également de la chaleur au milieu. Cette énergie calorifique, localisée à la surface, va se dissiper par conduction thermique. Le processus de recombinaison dépend des propriétés particulières du matériau absorbant, il peut se faire sous quatre formes : par transition interbande d'électrons avec émission de photons, par porteurs libres, l'énergie cédée servant à accroître leur agitation thermique, par le réseau. avec émission de phonons acoustiques, par les impuretés et défauts, par recombinaison indirecte par l'intermédiaire des niveaux contenus dans la bande interdite.

Les deux premiers mécanismes sont dominants tant pour les lasers pulsés (rubis, YAG) que continus (Argon). Pour les lasers à argon $(\lambda=0,53 \mu \mathrm{m})$ et à 
rubis $(\lambda=0,69 \mu \mathrm{m})$, l'absorption par porteurs libres sera moins importante que celle bande à bande, alors que c'est le contraire lorsque la longueur d'onde du laser se rapproche de l'énergie de la bande interdite $\mathrm{du}$ semiconducteur (YAG par exemple, $\lambda=1,06 \mu \mathrm{m}$ ).

Mentionnons que pour les lasers continus à longueur d'onde élevée, tels que le laser $\mathrm{CO}_{2}$ à $10,6 \mu \mathrm{m}$, l'absorption se fera essentiellement par les impuretés ou les défauts, du moins au voisinage de la température ambiante.

Pour terminer, notons que lorsque l'irradiation laser est intense (par exemple $1 \mathrm{~J} / \mathrm{cm}^{2}$ pendant $25 \mathrm{~ns}$ pour un laser à rubis), la concentration $\Delta R$ d'électrons photogénérés devient très forte : de l'ordre de $10^{20} \mathrm{~cm}^{-3}$ en considérant un coefficient d'absorption de $10^{5} \mathrm{~cm}^{-1}$. Il en résulte une densité de charges dans la région superficielle très importante. La recombinaison Auger devient alors beaucoup plus probable que toutes les autres transitions, de type bande à bande ou par les niveaux d'impuretés.

1.2 EVAluation DES TEMPÉRATURES ATTEINTES. Nous avons vu que la lumière émise par le laser est absorbée par excitation d'électrons dans une zone proche de la surface du semiconducteur, leur excès d'énergie se thermalise très rapidement, avec une constante de temps de l'ordre de $10^{-12} \mathrm{~s}$ [22] pour être finalement transformée en chaleur qui se propagera par conduction thermique avec une constante de diffusion de l'ordre de $10^{-10} \mathrm{~s}$, très inférieure à la durée (typiquement 10-100 ns) des impulsions laser. Pour connaître les températures atteintes durant l'irradiation, il est alors juste nécessaire de suivre la dépendance temporelle de l'équation de propagation de la chaleur avec un terme de génération correspondant à l'énergie déposée par le laser.

1.2.1 Résolution de l'équation de propagation de la chaleur. - Dans le cas le plus simple d'une surface plane exposée à une irradiation en incidence normale, la distribution en température en profondeur est obtenue en résolvant l'équation unidimensionnelle de propagation de chaleur, qui est de la forme :

$$
C_{\mathrm{S}} \rho \frac{\partial T}{\partial t}=I(x, t) \alpha(x)+\frac{\partial}{\partial x}\left(k \frac{\partial T}{\partial x}\right)
$$

où $C_{\mathrm{S}}$ désigne la chaleur spécifique du milieu absorbant, $\rho$ sa densité, $T$ la température à chaque instant $t$ ou à chaque abscisse $x, k$ la conductivité thermique, $\alpha(x)$ le coefficient d'absorption de la lumière laser.

Une solution analytique de cette équation pourrait être trouvée si tous les paramètres $\alpha, k, \rho$ étaient constants en fonction de $T$; dans la réalité il n'en est rien et il est nécessaire de faire certaines hypothèses quant à la dépendance en température de ces paramètres. Plusieurs calculs des distributions de température ont été publiés dans la littérature [23-36] qui diffèrent essentiellement les uns des autres par les choix des valeurs numériques des différents paramètres, ainsi que par leur évolution avec $T$.
1.2.2 Discussion et comparaison des résultats. Pour des lasers pulsés, les divers auteurs considèrent une impulsion soit rectangulaire, soit gaussienne. La plupart des calculs ont été effectués pour des lasers à rubis [23-31], mais aussi YAG [29-32] avec ou sans doubleur de fréquence, irradiant le silicium, le germanium [25] ou le tellurure de cadmium [30].

Pour des lasers continus (argon par exemple), on considère que l'irradiation d'un point varie brutalement (impulsion rectangulaire) [33-35], un modèle plus complexe a été proposé [36] qui tient compte d'un éclairement gaussien radial.

Comme indiqué précédemment, le choix des valeurs numériques des divers paramètres est essentiel : cela est illustré par la figure 1 où est reportée la variation de la température en fonction du temps en négligeant la variation avec $T$ des paramètres, sauf celle de la conductivité thermique $k$.

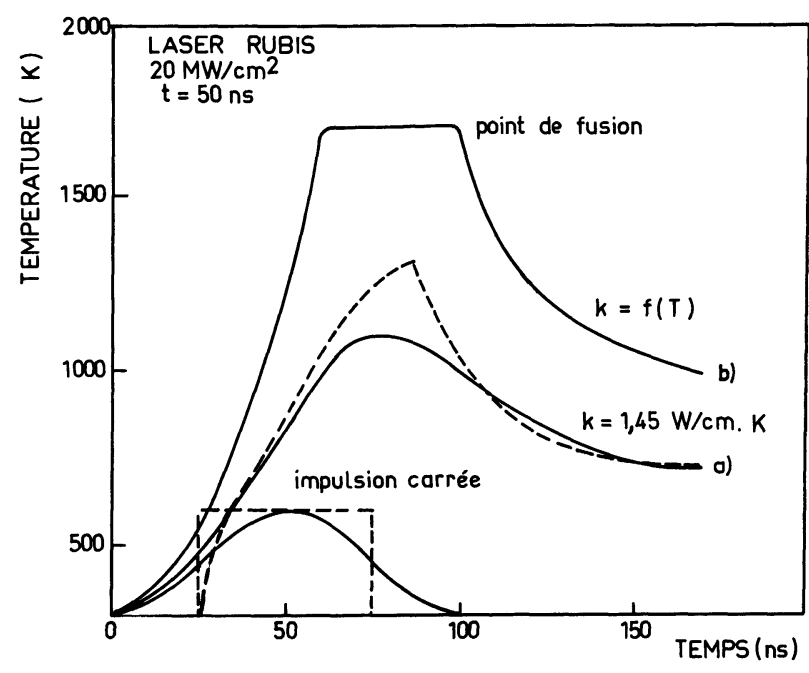

Fig. 1. - Calculs de la température atteinte en surface pour du silicium amorphe en fonction du temps. Les calculs ont été menés pour une impulsion délivrée par un laser Rubis, de densité $1,0 \mathrm{~J} / \mathrm{cm}^{2}$, de durée $50 \mathrm{~ns}$, de forme carrée (en pointillés) ou gaussienne (trait plein) en prenant le coefficient de conductivité thermique. a) Constant $k=1,45 \mathrm{~W} / \mathrm{cm} \mathrm{K}$. b) Variable en fonction de la température [25]

[Calculated temperature of the surface layer of amorphous silicon $v s$ time for an energy density of $1.0 \mathrm{~J} / \mathrm{cm}^{2}$, a pulse duration of 50 ns with a flat (dashed line) or Gaussian (full line) laser pulse shape and by taking for the thermal conductivity constant : either a constant value of $k=1.45 \mathrm{~W} / \mathrm{cm} \mathrm{K}$ or a $k$ value changing with temperature [25].]

La figure 2 montre l'évolution de la température en fonction du temps $t$ à la surface et à différentes profondeurs d'un silicium vierge éclairé par ủne impulsion gaussienne de durée (mi-puissance) $25 \mathrm{~ns}$ de $1 \mathrm{~J} / \mathrm{cm}^{2}$ (40 MW/ $\mathrm{cm}^{2}$ ) [30]. On constate que la température au voisinage de la surface croît très rapidement, jusqu'à ce que l'énergie incidente approche de sa valeur maximale, puis lorsque la température de fusion est atteinte, l'évolution de $T$ est temporairement stoppée jusqu'à ce que la zone de surface soit entièrement fondue. A ce moment, la réflectivité 


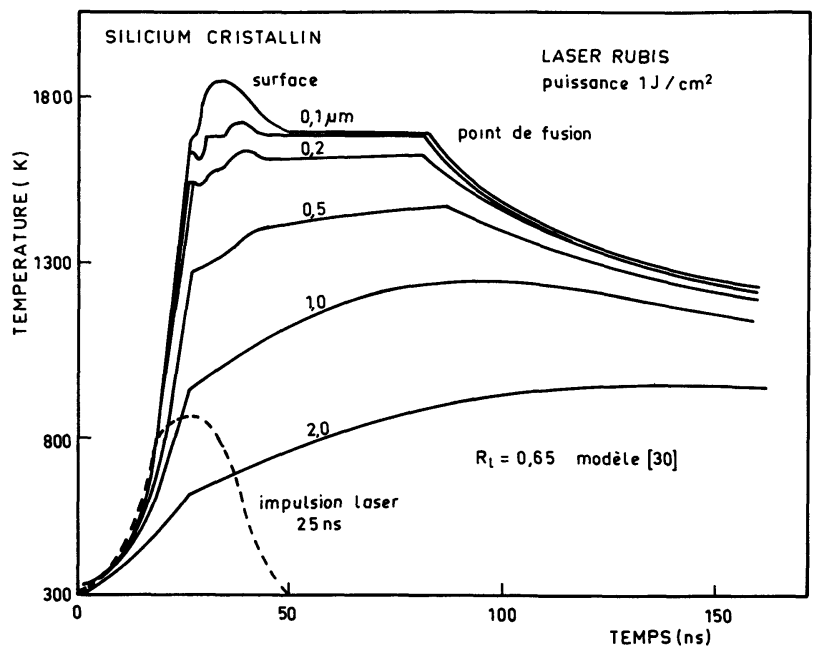

Fig. 2. - Calculs de la température atteinte en surface et à différentes profondeurs dans le silicium cristallin en fonction du temps. Les calculs ont été menés pour une impulsion gaussienne de $25 \mathrm{~ns}$, d'énergie $1 \mathrm{~J} / \mathrm{cm}^{2}$, délivrée par un laser à rubis [30].

[Predicted time dependence of temperature at different depths of virgin cristalline silicon irradiated by a ruby laser pulse of $25 \mathrm{~ns}$ and $1.0 \mathrm{~J} / \mathrm{cm}^{2}$ energy density [30].]

du matériau croît de sorte que la puissance absorbée diminue et qu'à une centaine d'angströms de la surface, le chauffage ne se fait plus par absorption mais seulement par conduction, ceci explique la diminution de température constatée entre 0,1 et $0,2 \mu \mathrm{m}$.

Après la fusion complète de la surface, la température croît de nouveau, mais avec une vitesse inférieure, due à l'augmentation de la conductivité dans la phase liquide. Ensuite la température retombe à un niveau correspondant à la recristallisation, se maintient ainsi un temps relativement long (30 ns), puis retombe rapidement.

Des courbes analogues sont calculées pour le silicium amorphe [30], nous donnons quelques exemples sur la figure 3 .

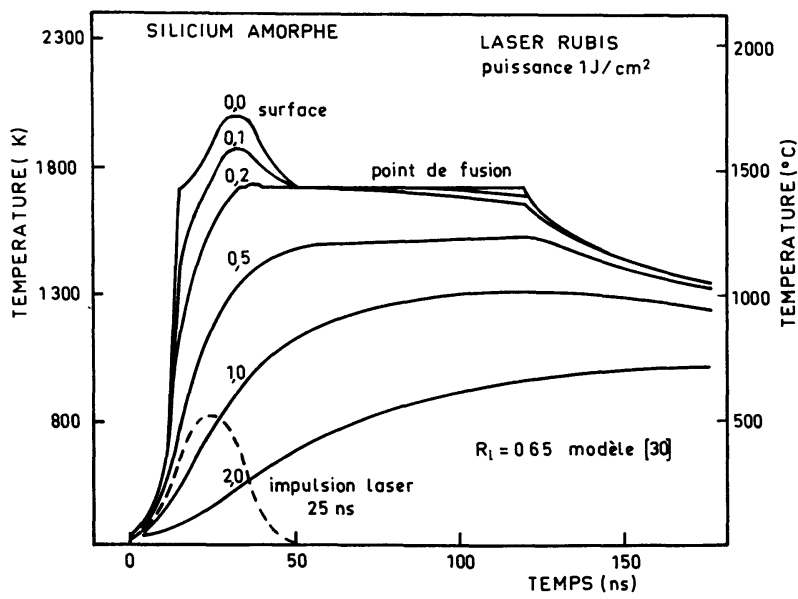

Fig. 3. - Calculs analogues à la figure 2 pour du silicium amorphe [30].

[Same calculations as for figure 2 but for an amorphous silicon layer [30].]
Notons que d'autres représentations ont été utilisées dans la littérature : évolution de l'épaisseur fondue en fonction du temps, en fonction de la puissance laser [25-29], ou encore température atteinte en fonction de la profondeur pour différentes énergies laser [26] ou pour différents temps [30].

Dans la pratique, la représentation la plus utile est celle donnant l'énergie laser nécessaire pour fondre une épaisseur donnée de silicium (Fig. 4). Nous avons regroupé sur cette figure les différents modèles publiés et nous pouvons constater que, pour une énergie donnée, l'épaisseur calculée pour la zone fondue peut varier très notablement. Nous pouvons observer que la valeur attribuée à la réflectivité de la zone liquide est le paramètre le plus sensible, d'où l'intérêt de la mesurer expérimentalement en fonction du temps au cours de l'irradiation; nous reviendrons sur cette expérience par la suite.

Les calculs qui ont été menés pour déterminer la température atteinte, bien que donnant des valeurs pouvant dépasser celle de fusion, ne constituent pas une preuve que la fusion est effective. Pour des temps de fusion aussi courts, certains auteurs préfèrent considérer l'existence d'un plasma très dense ou d'onde de choc laser. La recristallisation observée expérimentalement ne serait alors plus une simple épitaxie en phase liquide, mais s'opérerait suivant un schéma plus complexe qui aboutirait aux mêmes résultats [1]; dans ce modèle, les lacunes jouent un rôle prédominant, leur très grande mobilité à haute température $\left(\simeq 10^{-4} \mathrm{~cm}^{2} / \mathrm{s}\right)$ leur permet de parcourir en 50-100 ns des distances suffisantes pour réarranger un cristal perturbé.

Par ailleurs, il ne faut pas perdre de vue que ces processus, au cours de l'irradiation laser, se font sous une très forte photo- et thermo-ionisation, stimulée

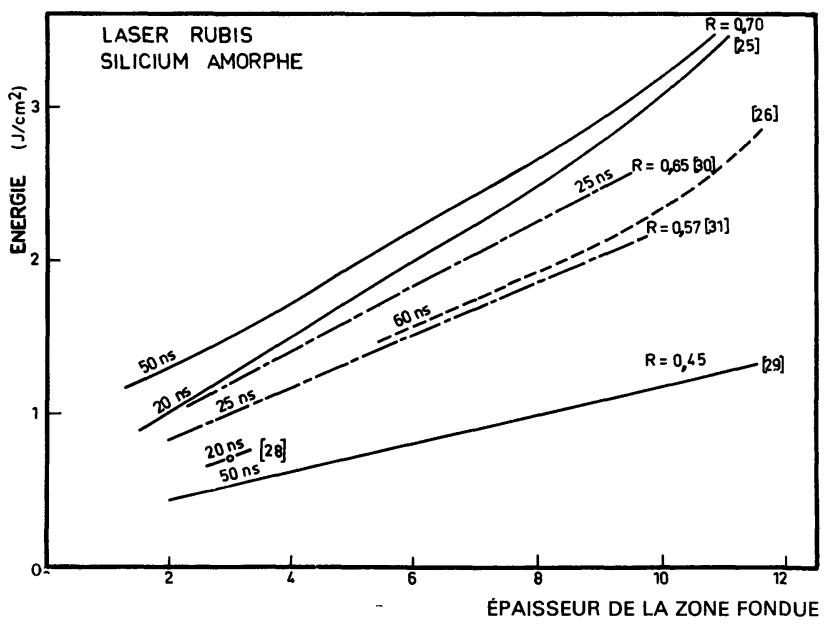

Fig. 4. - Calcul de l'évolution de l'épaisseur de silicium amorphe fondu sous une impulsion d'un laser à rubis en fonction de sa puissance, de sa durée et pour différentes valeurs du coefficient de réflexion.

[Theoretical evolution of the thickness of molten amorphous silicon under ruby laser illumination $v s$. power density, pulse duration and for various reflection coefficients.] 
par la présence d'un puissant champ électrique qui peut atteindre $5 \times 10^{4} \mathrm{~V} / \mathrm{cm}$, ce qui facilite la transformation du système en un état thermodynamiquement supersaturé et instable. Pour le moment, il est difficile de trancher définitivement la question du mécanisme microscopique réel.

\subsection{NOUVEL ÉTAT STABLE : STRUCTURE CRISTALLINE-} DOPANTS. - La plupart des travaux sur le recuit laser de couches superficielles perturbées ou amorphes publiés à ce jour portent sur des résultats expérimentaux obtenus en l'absence d'étude théorique. Ceci explique peut-être la grande variété des puissances laser utilisées et certaines divergences dans les conclusions des auteurs. Essentiellement, deux régimes très différents d'emploi sont possibles : épitaxie solide ou liquide. Deux effets de l'irradiation laser sont particulièrement intéressants, nous les considérerons successivement.

a) Epitaxie en phase liquide. - 1.3.1 Recristallisation de couches perturbées. - Essentiellement deux méthodes ont été employées pour caractériser la structure cristalline après recuit laser : la rétrodiffusion de particules chargées et la microscopie électronique, bien que des expériences de spectroscopie Raman [38] et de rayons $X$ [39] aient été employées quelquefois. $\mathrm{La}$ rétrodiffusion de particules dont l'énergie est de l'ordre du MeV (diffusion Rutherford) [40] permet de mesurer à la fois le degré d'amorphisation de la surface perturbée, les profils de concentration des défauts et de certaines impuretés. Cette technique a permis de montrer dès le début des travaux la qualité des couches recuites lorsque la phase liquide est atteinte [41]. Un

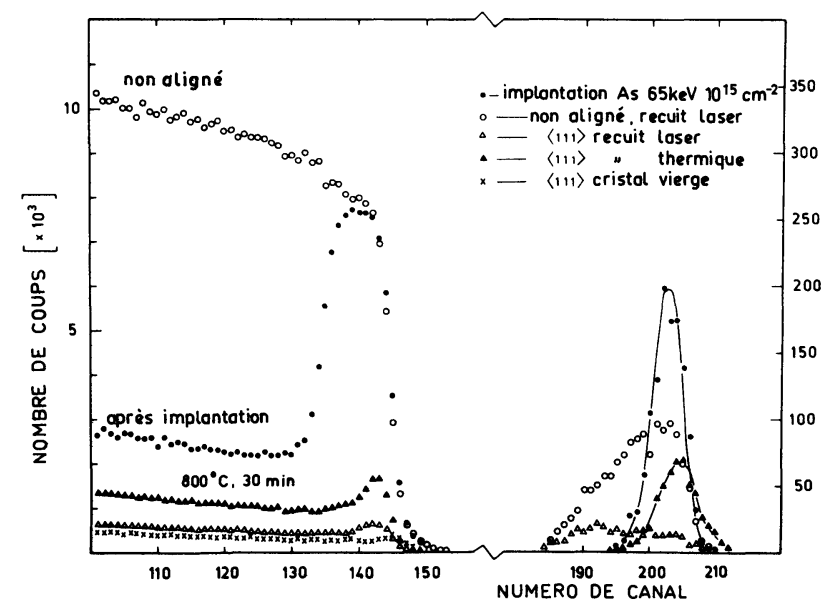

Fig. 5. - Spectres RBS en et hors conditions de canalisation (axe 111) pour des ions ${ }^{4} \mathrm{He}^{+}$d'énergie $1,2 \mathrm{MeV}$ tombant sur une cible de silicium préalablement implantée par des ions $\mathrm{As}^{+}$de $65 \mathrm{keV}\left(10^{15} \mathrm{~cm}^{-2}\right)$ et recuit par laser. Les résultats pour un recuit thermique conventionnel $\left(800^{\circ} \mathrm{C}, 30 \mathrm{~min}\right)$ sont également reportés [41].

[RBS spectra in and off-channelling conditions for ${ }^{4} \mathrm{He}^{+}$ion $s$ of $1.2 \mathrm{MeV}$ impinging along the $\langle 111\rangle$ axis a silicon crystal, which has been previously implanted by $\mathrm{As}^{+}\left(65 \mathrm{keV}, 10^{15} \mathrm{~cm}^{-2}\right)$ and laser annealed. Results obtained after conventional thermal annealing $\left(800{ }^{\circ} \mathrm{C}, 30 \mathrm{~min}\right.$.) are also reported.] exemple de spectre obtenu avant et après recuit d'une couche perturbée est donné sur la figure 5. Notons que cette technique d'analyse a un seuil de sensibilité entre $1 \%$ et $1 \%$ d'atomes déplacés à plus de $0,2 \AA$ de leur site cristallin habituel, suivant les conditions expérimentales.

- Vitesse de recristallisation. - Il a été possible de recristalliser une couche amorphe d'épaisseur $4000 \AA$, formée par bombardement ionique, pendant la durée d'une seule impulsion laser, soit en $10^{-7} \mathrm{~s}$ environ, ce qui correspond à une vitesse de recristallisation de $4 \times 10^{10} \AA / \mathrm{s}$, alors que la vitesse maximale en phase solide à $1400^{\circ} \mathrm{C}$ ne serait que de $2 \times 10^{7} \AA / \mathrm{s}$. Cette constatation est évidemment en faveur du modèle de recristallisation par simple épitaxie liquide.

- Homo et hétérostructure. - Jusqu'ici nous n'avons considéré que la recristallisation de couches superficielles d'un monocristal perturbé par bombardement ionique. Plusieurs auteurs [53-57] ont montré que des films amorphes évaporés à la surface d'un monocristal sous vide conventionnel $\left(\simeq 10^{-6}\right.$ torr $)$ et sans préparation sophistiquée de la surface, pouvaient également être recristallisés. Ce résultat ouvre des perspectives nouvelles dans la réalisation de couches épitaxiales.

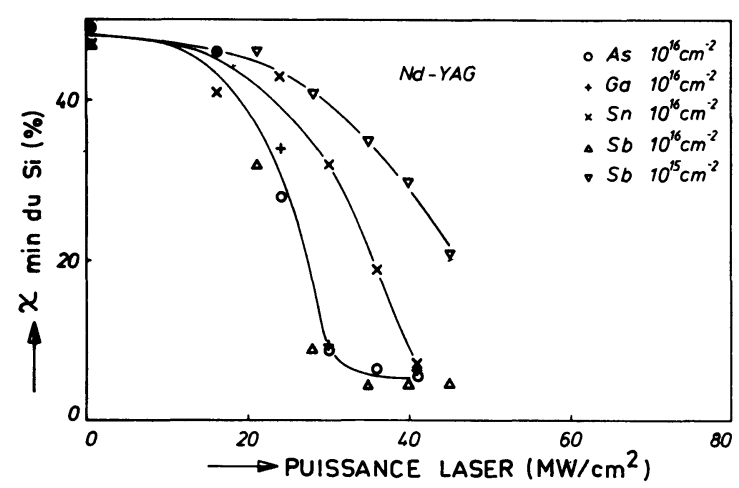

o)

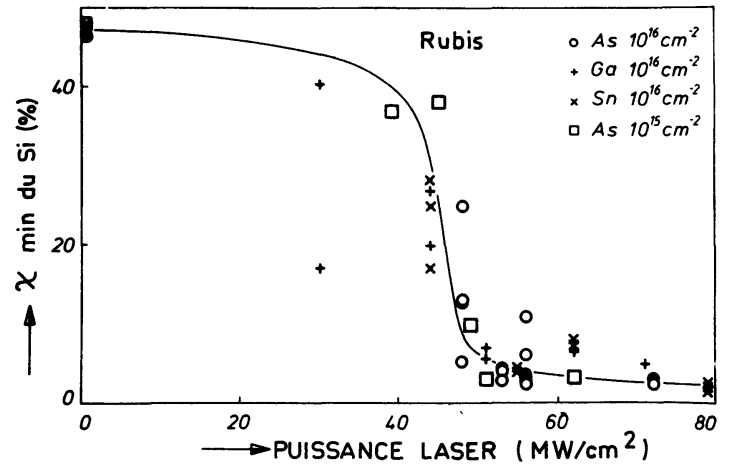

Fig. 6. - Mise en évidence d'un seuil de puissance laser pour la recristallisation : évolution de l'atténuation du rendement de rétrodiffusion en conditions de canalisation pour différents ions implantés en fonction de la puissance laser, respectivement pour une impulsion Nd : YAG de 180 ns (Fig. a) et pour une impulsion rubis de 25 ns [59].

[Threshold for laser regrowth reported for $\mathrm{Nd}$ : YAG (Fig. a) and rubis lasers (Fig. b) after implantation of various dopants into silicon. RBS minimum yield $\chi_{\min }$ is the parameter measured.] 
- Longueur d'onde du laser. En amorphisant une couche de silicium monocristalline sur une épaisseur de $2000 \AA$ à l'aide d'ions $\mathrm{As}^{+}, \mathrm{Ga}^{+}, \mathrm{Sn}^{+}$et $\mathrm{Sb}^{+}$ d'énergie convenable, on [58-59] a pu montrer que le seuil de recristallisation était indépendant de la nature de l'ion implanté (Fig. 6) lorsqu'un laser à rubis est employé. Par contre, pour un laser YAG, ce seuil dépend à la fois de la nature de l'ion et de sa dose (Fig. 6) : les porteurs libres interviennent fortement dans l'absorption.

La bonne recristallisation des couches au-dessus du seuil a été confirmée par microscopie électronique par plusieurs auteurs [44, 48, 60-62] (Fig. 7). Par contre. pour des surfaces ayant été implantées à des doses dépassant $10^{16} \mathrm{~B}^{+} / \mathrm{cm}^{2}$ et recuites par un laser à rubis. on [64] a constaté par réflexion Bragg de rayons $X$ des contractions unidimensionnelles du réseau de silicium.

$\mathrm{Au}$ voisinage du seuil de fusion, des zones polycristallines ont été mises en évidence [64-65] tout près de l'interface cristal-amorphe, montrant une recristallisation incomplète. Il convient donc de déterminer pour chaque cas la puissance laser, qui est fonction également de la longueur d'onde et de la durée de l'impulsion lumineuse, du nombre d'impulsions employé, en plus des propriétés optiques spécifiques à chaque silicium. A titre d'exemple, nous reportons sur le tableau I les résultats obtenus [66] avec un laser à rubis sur des monocristaux dopés au phosphore comportant des précipités. Il est évident qu'un matériau plus ou moins dégradé par bombardement aurait des propriétés optiques différentes, donc des énergies optimales également différentes.

Tableau I. - Epaisseur recristallisée par un laser à rubis illuminant un monocristal de silicium fortement dopé ayant des précipités [66].

$\begin{array}{cccc}\begin{array}{c}\text { Densité } \\ \text { d'énergie } \\ \mathrm{J} / \mathrm{cm}^{2}\end{array} & \begin{array}{c}\text { Durée } \\ \text { de l'impulsion } \\ -\end{array} & \begin{array}{c}\text { Nombre } \\ \text { ns }\end{array} & \begin{array}{c}\text { Epaisseur } \\ \text { recuite } \\ \text { d'impulsions }\end{array} \\ \begin{array}{c}\mu \mathrm{m} \\ 1,5\end{array} & 50 & - & - \\ 1,5 & 50 & 1 & 0,29 \\ 1,5 & 50 & 2 & 0,38 \\ 1,5 & 50 & 5 & 0,56 \\ 2 & 20 & 7 & 0,58 \\ 2 & 20 & 1 & 0,57 \\ 2 & 20 & 3 & 0,70 \\ 2 & 20 & 5 & 0,75 \\ 3 & 20 & 14 & 0,77 \\ & & 1 & 0,95\end{array}$

Fig. 7. - Comparaison de l'effet de recuit thermique et laser sur du silicium implanté par des ions ${ }^{11} \mathrm{~B}^{+}\left(35 \mathrm{keV}, 3 \times 10^{15} \mathrm{~cm}^{-2}\right)$ observé au microscope électronique à transmission : recuit laser $1,7 \mathrm{~J}$ (Fig. $a$ ) ; recuit thermique $\left(900^{\circ} \mathrm{C}, 30 \mathrm{~min}\right.$.) (Fig. $b$ ); recuit thermique $\left(1100^{\circ} \mathrm{C}, 30 \mathrm{~min}\right.$ ) (Fig. c) $[152,153]$.

[Observation by TEM of a silicon crystal implanted by ${ }^{11} \mathrm{~B}^{+}$ $\left(35 \mathrm{keV}, 3 \times 10^{15} \mathrm{~cm}^{-2}\right.$ ) after laser annealing (1.7 J) (Fig. a) ; thermal annealing for $30 \mathrm{~min}$. at $900^{\circ} \mathrm{C}$ (Fig. b) and $1100^{\circ} \mathrm{C}$ (Fig. c).]

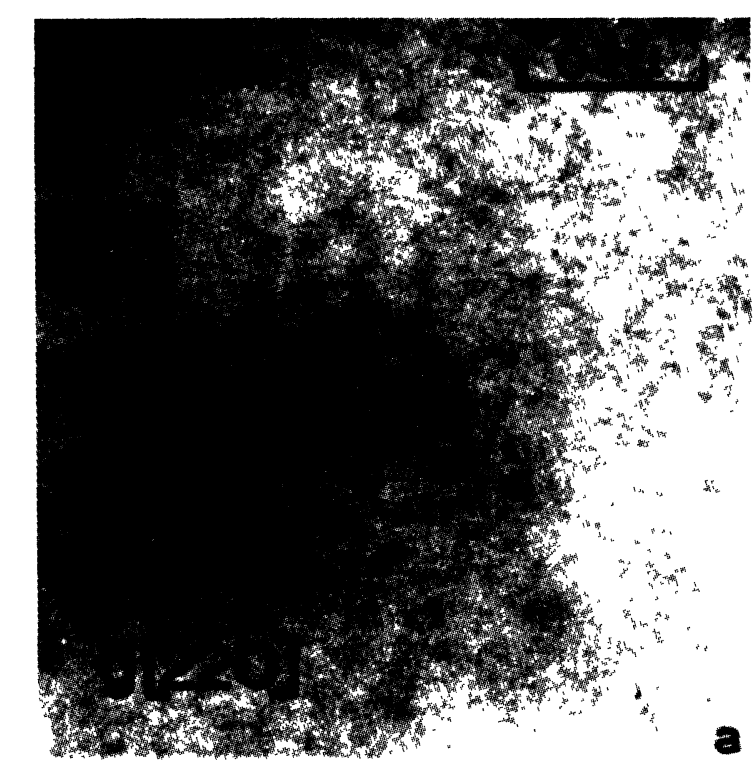

b)

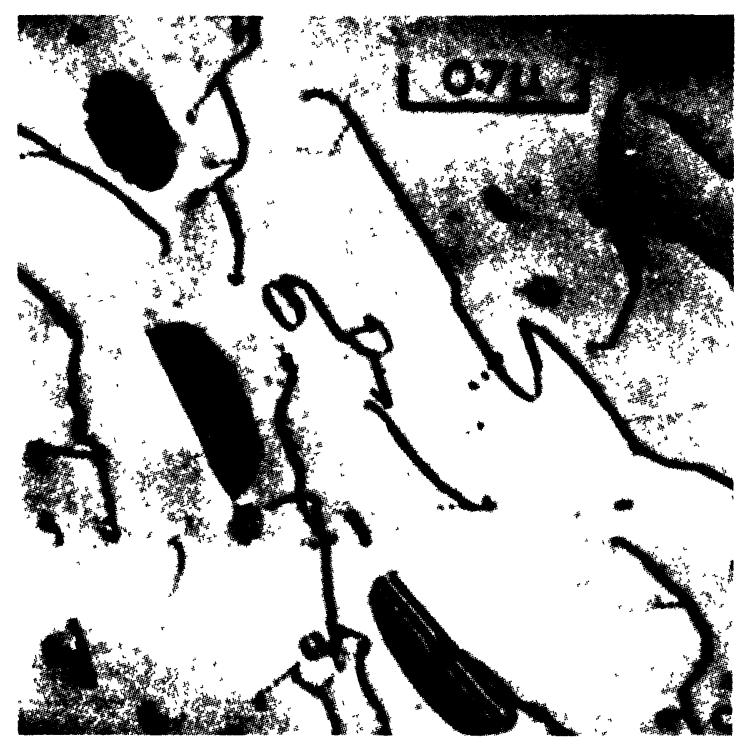

c)

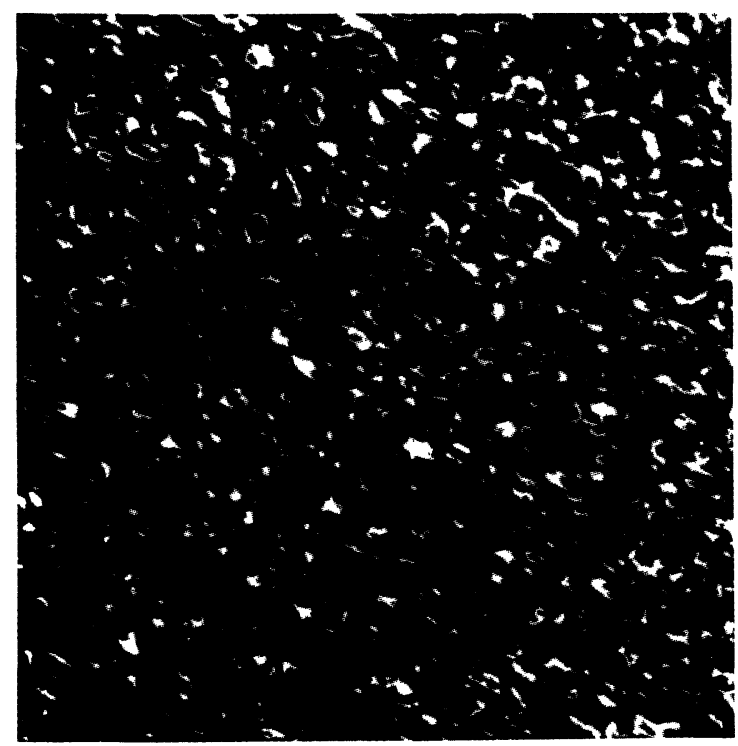


En comparant les valeurs de l'épaisseur recuite à celles calculées plus haut (Fig. 4), on constate que pour une impulsion unique, un assez bon accord est obtenu pour un coefficient de réflexion de $R=0,70$ avec le modèle [25]. Cependant, lorsqu'on accroît le nombre de tirs, les zones recristallisées augmentent jusqu'à une limite, assez proche de la valeur calculée à partir du coefficient de réflexion mesuré expérimentalement. Ceci est dû à la modification des propriétés optiques, très sensibles à la qualité du matériau.

1.3.2 Redistribution des dopants. - Lorsque la densité d'énergie est suffisante pour produire la fusion en surface du silicium, s'il est dopé, on observera une complète redistribution des impuretés dans le liquide par suite du coefficient de diffusion très élevé (Tableau II) [67] des impuretés dans le silicium fondu.

Tableau II. - Coefficient de diffusion des dopants dans le silicium fondu $\left(\mathrm{cm}^{2} / \mathrm{s}\right)[67]$.

\begin{tabular}{ccccc} 
B & P & Sb & Ga & In \\
\hline$-1,3 \times 10^{-4}$ & $2,7 \times 10^{-4}$ & $1,4 \times 10^{-4}$ & $6,6 \times 10^{-5}$ & $1,7 \times 10^{-5}$
\end{tabular}

Très rapidement $(\simeq 100 \mathrm{~ns})$, la solidification intervient à partir de l'interface solide cristallin-liquide à une vitesse très élevée de l'ordre de $1-2 \mathrm{~m} / \mathrm{s}$. Pour ces vitesses élevées, les coefficients de ségrégation effectifs devraient être proches de l'unité. Toutefois, pour des films minces [68], le coefficient réel peut être inférieur.

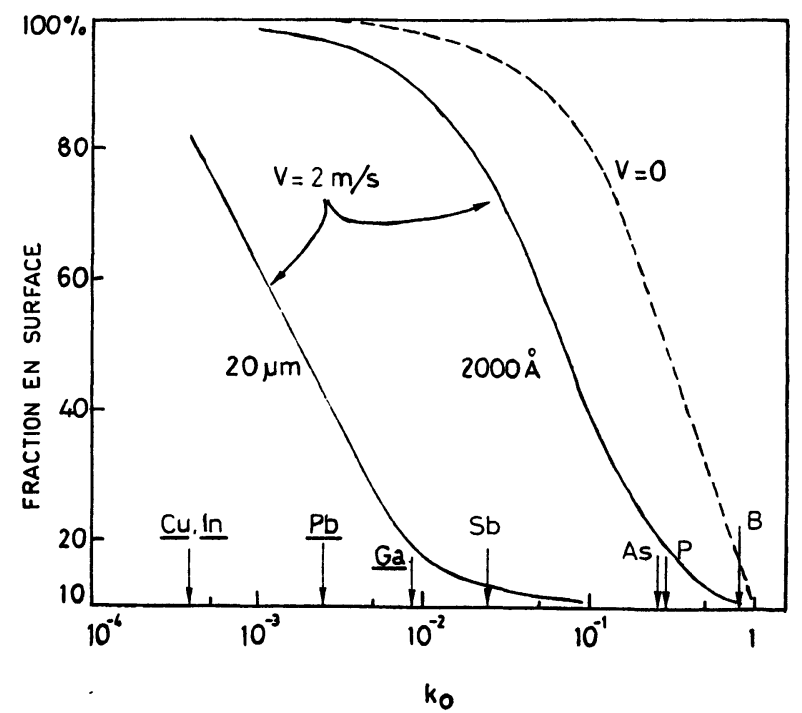

Fig. 8. - Fraction d'impuretés accumulées en surface dans une zone correspondant à $10 \%$ de la zone totale fondue en fonction du coefficient de partage à l'équilibre, pour différentes épaisseurs de films et pour deux vitesses $V$ de recristallisation [128].

[Fraction of impurities segregated in the outer most $10 \%$ of a liquid layer as a function of the equilibrium distribution coefficient. Two thickness and two regrowth speeds are reported.]

La figure 8 représente la fraction de dopants calculée se retrouvant dans les $10 \%$ superficiels de la couche fondue en fonction du coefficient de ségrégation. La figure 9 donne les résultats expérimentaux pour la plupart des dopants implantés après recuit laser. o)
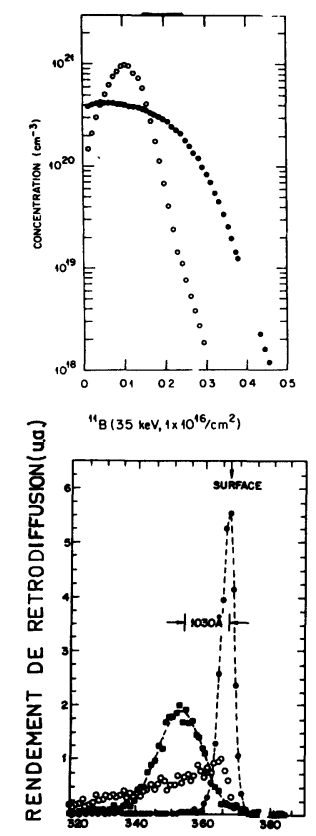

Cu(150keV $\left.5.10^{15} / \mathrm{cm}^{2}\right)$
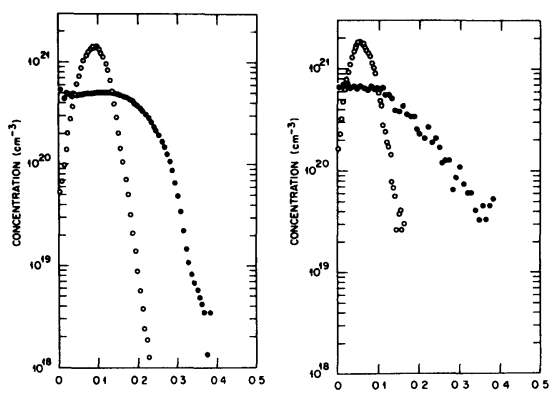

${ }^{31} \mathrm{P}\left(80 \mathrm{keV} 116 \times 10^{\left.16 / \mathrm{cm}^{2}\right)}\right.$

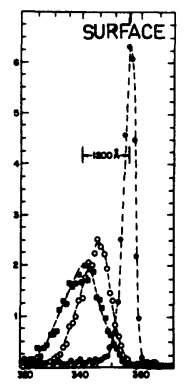

$75_{\text {AS }}\left(100 \mathrm{keV}, 14 \times 10^{16} / \mathrm{cm}^{2}\right)$

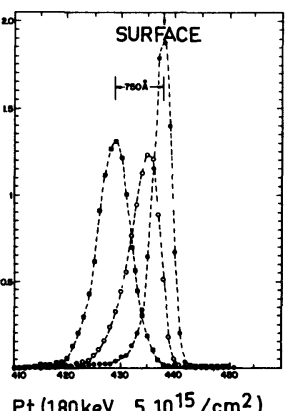

A nalyse R.B.S

- après implantation

- recuit thermique

$1000^{\circ} \mathrm{C} 30 \mathrm{~min}$

- recuit laser Nd YAG $1,06 \mu \mathrm{m}$ $50-100 \mathrm{MW} / \mathrm{cm}^{2}$

[155]

Fig. 9. - Influence d'un recuit laser en phase liquide. a) Sur les profils (SIMS) d'éléments dopants ayant un coefficient de ségrégation proche de $1[154,155]$; b) Sur la distribution (RBS) des impuretés ayant des coefficients de ségrégation faibles [156].

[Liquid phase laser annealing : effect on dopant distribution. a) SIMS profiling of various dopants having a segregation coefficient close to one ; $b$ ) RBS analysis for dopants having small segregation coefficients.] 
b) Epitaxie en phase solide. - 1.3.3 Recristallisation. - Plusieurs auteurs ont trouvé que pour des puissances laser trop faibles pour atteindre la fusion du silicium, la recristallisation s'opérait par épitaxie en phase solide, d'une manière similaire à ce qui se passe au cours de recuits thermiques conventionnels. Rappelons que dans ce dernier cas, la recristallisation d'un film amorphe déposé sur du silicium $\langle 100\rangle$ se fait à la vitesse

$$
v=v_{0} \exp \left(-\frac{E_{\mathrm{a}}}{k T}\right)
$$

avec $v_{0} \simeq 2,9 \times 10^{9} \mathrm{~cm} / \mathrm{s}$ et $E_{\mathrm{a}}=2,3-2,7 \mathrm{eV}$. Pour des orientations cristallines différentes, des vitesses de recristallisation sont plus faibles d'un facteur $5-10$, ce qui a été confirmé également pour recuit laser [69]. Le temps nécessaire à recristalliser un film d'une épaisseur donnée est évidemment fonction de la température atteinte. A titre d'exemple, la figure 10 représente le temps mis pour un film d'épaisseur $500 \AA$ [70].

1.3.4 Redistribution des dopants. - En dessous du seuil d'apparition de la zone fondue, la redistribution des dopants est généralement négligeable par suite du coefficient de diffusion très faible $\left(\simeq 10^{-10}\right.$. $\left.10^{-11} \mathrm{~cm}^{2} / \mathrm{s}\right)$ des impuretés en phase solide. Plusieurs auteurs [61-71-73] ont vérifié cette hypothèse. Toutefois, on a constaté pour une implantation d'arsenic dans le silicium [74-75], qu'en dessous du seuil, un étalement de la distribution apparaissait, qui ne peut

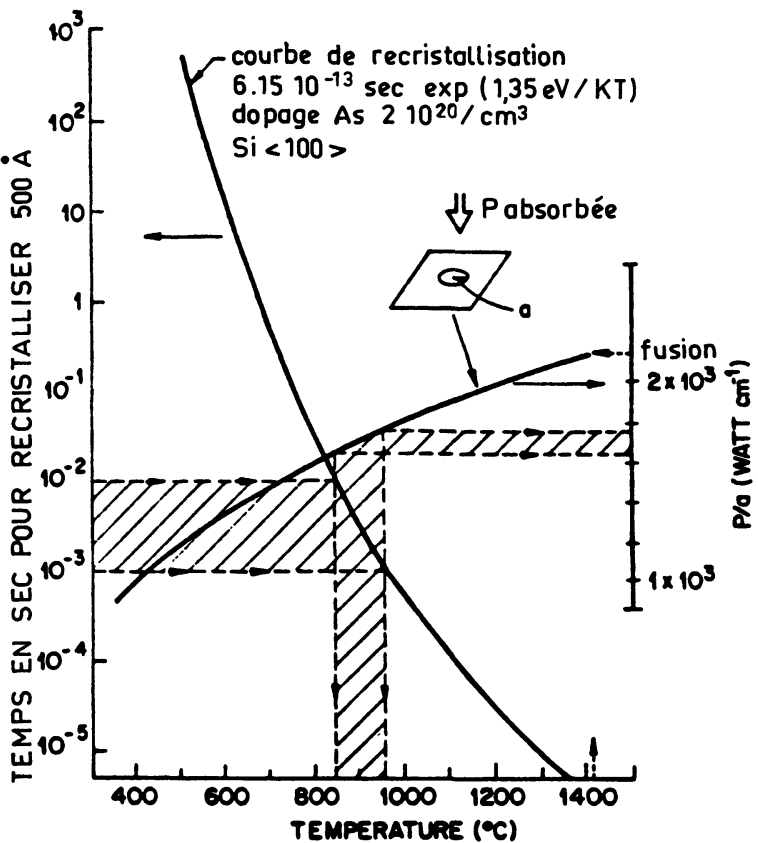

Fig. 10. - Temps nécessaire pour recristalliser $500 \AA$ de silicium amorphisé en fonction de la température du substrat. La puissance absorbée en fonction de la température atteinte au centre du spot est également reportée. Les zones hachurées définissent les régions de température et de puissance laser nécessaires pour recristalliser une couche amorphisée après implantation de $\mathrm{As}^{+}(30 \mathrm{keV}$, $\left.3 \times 10^{15} \mathrm{~cm}^{-2}\right)$ [157].

[Time to epitaxially regrow $500 \AA$ of amorphous silicon as a function of substrate temperature. Also plotted, the calculated absorbed laser power for spot radius as a function of steady state temperature attained at the center of the spot. The hatched areae define the temperature regions and laser powers necessary to regrow a surface bombarded by $\mathrm{As}^{+}$ions $\left(30 \mathrm{keV}, 3 \times 10^{15} \mathrm{~cm}^{-2}\right)$.]

Tableau III. - Défauts électriquement actifs observés après recuit laser de silicium (vierge ou implanté).

\begin{tabular}{|c|c|c|c|c|}
\hline Nature du substrat & Type de laser & Technique de mesure & Niveaux & Réf. \\
\hline Si type $\mathrm{N}$ implanté ${ }^{11} \mathrm{~B}^{+}$ & Rubis & TSC & $\begin{array}{l}E_{C}-0,11 \mathrm{eV} \\
E_{C}-0,16 \mathrm{eV} \\
E_{C}-0,22 \mathrm{eV} \\
E_{C}-0,34 \mathrm{eV} \\
E_{C}-0,40 \mathrm{eV} \\
E_{C}-0,51 \mathrm{eV}\end{array}$ & [76] \\
\hline Si Cz. Type P vierge & Rubis & DLTS & $\begin{array}{l}E_{v}+0,25 e V \\
E_{V}+0,37 e V\end{array}$ & [77] \\
\hline $\mathrm{Si}$ épitaxial type $\mathrm{N}$ implantation $\mathrm{Si}^{+}$ & Argon & DLTS & $\begin{array}{l}E_{C}-0,19 \mathrm{eV}(\mathrm{V}-\mathrm{O}) \\
\mathrm{E}_{\mathrm{C}}-0,28 \mathrm{eV} \\
\mathrm{E}_{\mathrm{C}}-0,49 \mathrm{eV} \\
\mathrm{E}_{\mathrm{C}}-0,56 \mathrm{eV}\end{array}$ & $\begin{array}{l}{[79]} \\
{[80]}\end{array}$ \\
\hline $\begin{array}{l}\text { Si FZ type NetP implanté } \mathrm{Sb}^{+}, \mathrm{As}^{+} \\
\mathrm{Si} \mathrm{Cz} \text { type } \mathrm{N} \text { et } \mathrm{P} \text { implanté } \mathrm{B}^{+} \\
\mathrm{Si} \text { vierge }\end{array}$ & YAG et $\mathrm{CO}_{2}$ & DLTS & $\begin{array}{l}E_{C}-0,10 \mathrm{eV} \\
E_{C}-0,18 \mathrm{eV}(\mathrm{V}-\mathrm{O}) \\
\mathrm{E}_{\mathrm{C}}-0,19 \mathrm{eV} \\
\mathrm{E}_{\mathrm{C}}-0,27 \mathrm{eV} \\
\mathrm{E}_{\mathrm{C}}-0,33 \mathrm{eV} \\
\mathrm{E}_{\mathrm{V}}+0,25 \mathrm{eV} \\
\mathrm{E}_{\mathrm{V}}+0,36 \mathrm{eV} \\
\mathrm{E}_{\mathrm{C}}-0,18 \mathrm{eV}(\mathrm{V}-\mathrm{O})\end{array}$ & [78] \\
\hline Silicium $\mathrm{Fz}$ type $\mathrm{N}$ implanté $\mathrm{PB}_{5}$ & Rubis & $\begin{array}{l}\text { TSC } \\
\text { EPR }\end{array}$ & $\begin{array}{l}E_{C}-0,18 \mathrm{eV}(\mathrm{V}-\mathrm{O}) \\
\mathrm{E}_{\mathrm{V}}+0,25 \mathrm{eV} \\
\mathrm{E}_{\mathrm{C}}-0,18 \mathrm{eV}(\mathrm{V}-\mathrm{O})\end{array}$ & $\begin{array}{l}{[31]} \\
{[82]}\end{array}$ \\
\hline
\end{tabular}


Tableau IV. - Comparaison recuits laser et thermique.

\begin{tabular}{|c|c|c|c|c|}
\hline & Mode de chauffage & Laser continu & Laser pulsé & Thermique classique \\
\hline $\begin{array}{l}\mathrm{H} \\
\mathrm{O} \\
\mathrm{M} \\
\mathrm{O}\end{array}$ & $\begin{array}{l}\text { Conditions typi- } \\
\text { ques }\end{array}$ & $\begin{array}{l}\text { Laser type } \mathrm{CW} \text { argon } \\
15-20 \mathrm{~W} \text { focalisé sur } \\
50 \mu \mathrm{m}^{2} \text {, durée d'ill. } \\
10^{-2}-10^{-3} \mathrm{~s}\end{array}$ & $\begin{array}{l}\text { Laser type rubis ou YAG- } \\
\mathrm{Nd} \text {, mono ou multimode, } \\
1-3 \mathrm{~J} / \mathrm{cm}^{2} \text {, impulsion durée } \\
25-100 \text { ns (mi-puissance) }\end{array}$ & Four conventionnel \\
\hline $\mathrm{E}$ & Mode de recristal- & & & \\
\hline$P$ & $\begin{array}{l}\text { lisation } \\
\text { Vitesse de recris- }\end{array}$ & Epitaxie solide & Epitaxie liquide & $\begin{array}{l}\text { Epitaxie solide } \\
15 \AA / s \text { a } 550 \mathrm{C}\end{array}$ \\
\hline $\begin{array}{l}\mathrm{I} \\
\mathrm{T}\end{array}$ & $\begin{array}{l}\text { Vitesse de recris- } \\
\text { tallisation }\end{array}$ & $10^{5} \AA / \mathrm{s}$ à $900^{\circ} \mathrm{C}$ & $1-2 \mathrm{~m} / \mathrm{s}$ & $\begin{array}{l}1,5 \mathrm{~A} / \mathrm{s} \text { a } 550^{\circ} \mathrm{C} \\
10^{7} \AA / \mathrm{s} \text { à } 1400^{\circ} \mathrm{C} \text { (estimé) }\end{array}$ \\
\hline $\begin{array}{l}\text { A } \\
\text { X } \\
\text { I }\end{array}$ & $\begin{array}{l}\text { Effet de l'orienta- } \\
\text { tion sur vitesse de } \\
\text { recristallisation }\end{array}$ & $\begin{array}{l}\text { Nettement meilleur sur } \\
\langle 100\rangle \text { que }\langle 111\rangle\end{array}$ & $\begin{array}{l}\text { Bon pour les deux orienta- } \\
\text { tions }\langle 100\rangle \text { et }\langle 111\rangle\end{array}$ & $\begin{array}{l}\text { Bon en }\langle 100\rangle \text {, médiocre } \\
\text { en }\langle 111\rangle\end{array}$ \\
\hline $\mathrm{E}$ & Effet sur impuretés & Peu sensible & Redistribution complète & Peu sensible \\
\hline $\begin{array}{l}\mathrm{H} \\
\mathrm{E} \\
\mathrm{T} \\
\mathrm{E} \\
\mathrm{R} \\
\mathrm{O}\end{array}$ & $\begin{array}{l}\text { Conditions cristal- } \\
\text { lisation }\end{array}$ & $\begin{array}{l}\text { Accroissement sensible } \\
\text { de la dimension des } \\
\text { grains (typiquement } \\
25 \mu)\end{array}$ & $\begin{array}{l}\text { Monocristallisation même } \\
\text { pour des films déposés sous } \\
\text { vide conventionnel (10E- } \\
6 \text { torr) sans préparation } \\
\text { spéciale du substrat }\end{array}$ & $\begin{array}{l}\text { Pas de monocristallisation } \\
\text { sauf pour films déposés } \\
\text { sous ultravide sur un cris- } \\
\text { tal convenablement pré- } \\
\text { paré }\end{array}$ \\
\hline
\end{tabular}

être interprété par la diffusion thermique conventionnelle, peut-être une diffusion accélérée induite par le laser est-elle possible?

1.4 Dommages RéSDUEls. - Bien que par microscopie électronique ou rétrodiffusion de particules chargées la recristallisation laser apparaisse quasi parfaite, des mesures électriques plus sensibles, généralement effectuées par TSC ou DLTS [31, 76-81], ont révélé la présence de défauts microscopiques pouvant être électriquement actifs. Un certain nombre de niveaux ont été trouvés dans la littérature (Tableau III), mais il nous semble qu'aucune conclusion définitive ne peut être tirée à l'heure actuelle car la nature des cristaux (CZ ou FZ) et la nature des dopants (certains implantés) n'étaient pas les mêmes. Dans le silicium vierge pur un seul niveau a été identifié à $E_{\mathrm{c}}-0,18 \mathrm{eV}$, correspondant à l'association lacune-oxygène $[\mathrm{V}-\mathrm{O}]^{-}$; il disparaît après un recuit thermique complémentaire à $500^{\circ} \mathrm{C}$ [31-82].

Pour résumer les caractéristiques essentielles $\mathrm{du}$ recuit laser, nous avons reporté sur le tableau IV les points essentiels en comparaison au recuit thermique conventionnel.

2. Techniques expérimentales. -2.1 CHOIX DU LASER. - Nous avons vu qu'il existe essentiellement deux régimes de recristallisation par épitaxie solide ou liquide. En utilisant un laser pulsé, seul le dernier domaine est possible car la durée d'impulsion est trop courte pour entraîner une guérison notable en phase solide. Par contre avec les lasers continus les deux modes de recuit sont possibles.

2.1.1 Lasers continus. - Kachurin [4], puis Gibbons [83], Auston [84] et Williams [70] ont successi- vement employé cette technique en focalisant le spot d'un laser continu de puissance $10-15 \mathrm{~W}$ sur des surfaces de $10-100 \mu \mathrm{m}^{2}$ avec un balayage du faisceau à une vitesse de l'ordre de $q q \mathrm{~cm} / \mathrm{s}$ et un déplacement de $q q$ dizaines de $\mu \mathrm{m} / \mathrm{s}$. Le schéma d'une installation expérimentale est représenté sur la figure 11. Le laser $\mathrm{CO}_{2}$ est également employé quelquefois [85-87]; dans ce cas l'absorption de la lumière ne se fait que par porteurs libres et varie très fortement avec la température [87].

LASER CONTINU

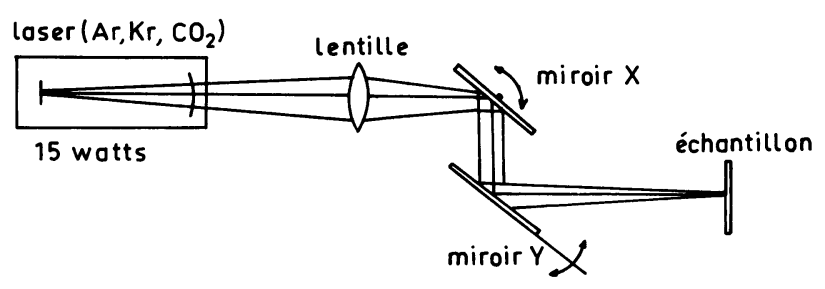

Fig. 11. - Schéma de principe d'un ensemble de recuit par laser continu avec focalisation et balayage du faisceau [158].

[Principle of the set-up used for continuous laser beam annealing with focus and scanning over the sample.]

2.1.2 Lasers pulsés. - Ce sont actuellement les plus employés, nous avons reporté sur la figure 12 les principaux types employés. On constate que la plupart des travaux font appel à des impulsions de durée $20-50 \mathrm{~ns}$. Avec les lasers ps $\left(10^{-12} \mathrm{~s}\right)$ des problèmes subsistent puisque du silicium cristallin est amorphisé au cours de l'éclairement [101]. Ce type de lasers n'entre donc pas dans le cadre de cet exposé. 


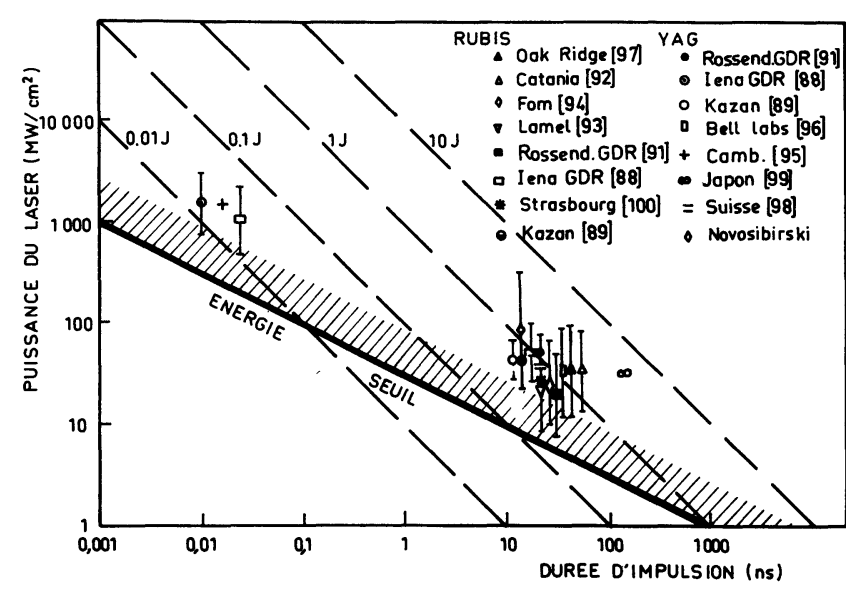

Fig. 12. - Domaines d'utilisation des lasers de puissance à impulsions (rubis ou YAG), utilisés par les principaux laboratoires en fonction des caractéristiques des impulsions. La zone hachurée correspond à l'énergie seuil.

[Domain of laser power density and pulse duration (for ruby and YAG) for annealing. The hatched zone corresponds to the threshold. The characteristics of the lasers used in the main laboratories are reported.]

$\mathrm{Au}$ début, la plupart des lasers fonctionnaient en multimode afin d'obtenir une puissance maximale. Cependant l'uniformité des faisceaux ainsi obtenus laissait à désirer, de sorte que de plus en plus, on fonctionne en régime monomode. Des modes de fonctionnement plus simples (en relaxé) $[5,11,102-$ 103] ou plus complexes [104-105] ont été également utilisés. Certains auteurs [1-41] ont proposé d'interposer des verres ou liquides diffuseurs pour uniformiser au mieux le faisceau. Les dimensions des spots dépendent des barreaux lasers, qui sont généralement de diamètre 6-30 $\mathrm{mm}$. Pour des aires inférieures à ces dimensions un seul tir suffit, alors que pour des dimensions supérieures un déplacement $x-y$ de l'échantillon peut s'avérer nécessaire. Dans ce cas, des problèmes peuvent se créer aux limites de chaque illumination, ils ont été considérés par Celler [87] (Fig. 13). Des ensembles automatisés, adaptés pour la production en chaîne, viennent d'apparaître sur le marché [106],

2.1.3 Technique annexe: mesure du temps de fusion. - Nous avons vu plus haut l'importance des divers paramètres sur le calcul des conditions de recuit. Le coefficient de réflexion, la durée de fusion peuvent être mesurés assez simplement [32, 107-110] à l'aide du dispositif schématisé sur la figure 14 : un laser continu (He-Ne par exemple) éclaire l'échantillon et la lumière réfléchie est mesurée à l'aide d'une photodiode après traversée d'un filtre sélectif. L'impulsion émise par le laser de puissance déclenche un oscilloscope à mémoire qui enregistre l'évolution temporelle de la lumière reçue. La sensibilité est suffisante pour distinguer une surface solide amorphe, cristalline ou liquide (Fig. 14).

3. Application à la technologie des photopiles. A ce jour, le recuit laser a été employé soit pour
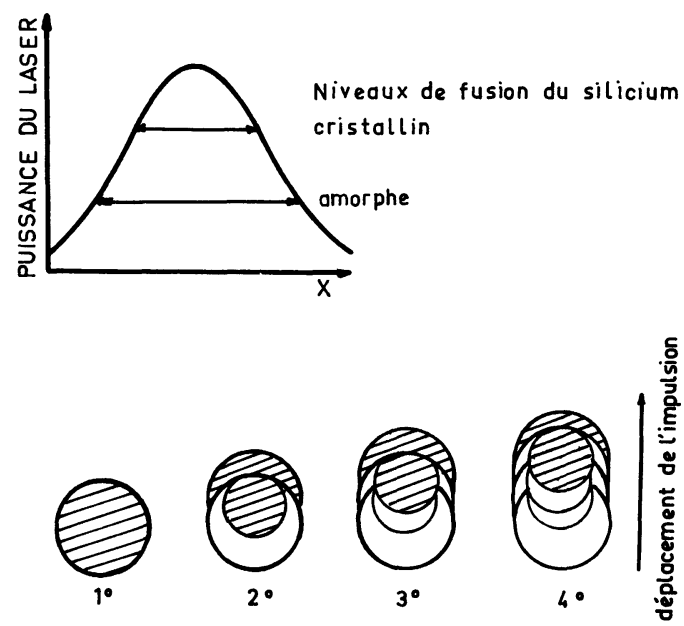

Fig. 13. - Représentation schématique de la fusion laser avec les seuils de fusion du silicium amorphe et cristallin. 1) Une première impulsion fond une large zone ; 2) Une deuxième impulsion fond une zone non encore exposée donc amorphe et une plus faible région dans la partie déjà recristallisée; 3 et 4) Progression de l'impulsion [87].

[Laser energy density as a function of position within a single laser spot. 1) First pulse produces large molten spot ; 2) Additional pulse melt grey area consisting of newly exposed amorphous silicon and smaller spot within previously recrystallized region; 3-4) Displacement of the regrown spots.]

MESURE DE LA REFLECTION OPTIOUE

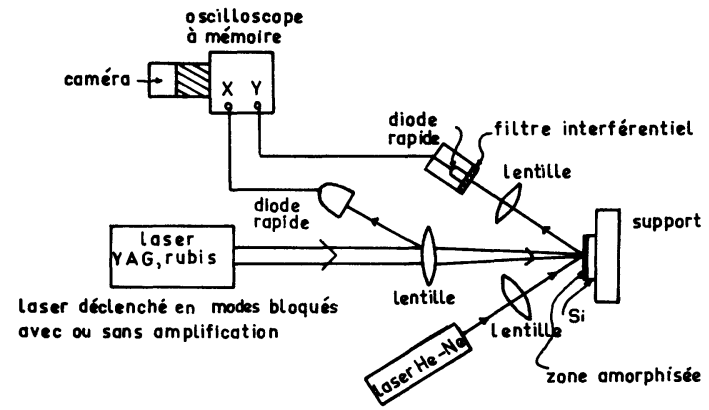

SIGNAL

nive ou 0

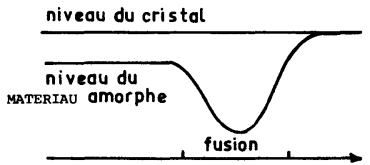

Fig. 14. - Schéma du montage expérimental permettant de mesurer le coefficient de réflection de la zone fondue. Les formes des signaux obtenus sont reportées dans le bas [109-110].

[Experimental arrangement used to measure the time resolved reflectivity of silicon during laser annealing.] 
préparer du silicium monocristallin ou polycristallin, soit en vue de la réalisation de la barrière de potentiel.

3.1 Elaboration DE SIL ICIUM. - Essentiellement deux méthodes ont été développées pour préparer soit des films cristallins autosupportés, soit des couches polycristallines déposées sur un substrat.

3.1.1 Cristallisation d'un ruban à partir d'un film polycristallin («ribbon to ribbon growth»). - Ce procédé développé par Motorola [111-113] comporte essentiellement deux étapes :

3.1.1.1 Dépôt de silicium sur un substrat convenable. - Il est bien connu qu'en déposant du silicium à partir d'une phase gazeuse (par exemple $\mathrm{SiH}_{4}$ ou $\mathrm{SiHCl}_{3}$ ) sur un substrat chauffé convenablement, on obtient des polycristaux de faibles dimensions sans orientation préférentielle (technique dite CVD) [114 119]. En choisissant correctement la nature du substrat, le film de silicium se détachera de son support au moment du refroidissement ("Thermal Expansion Shear Separation Technique ») par suite des coefficients de dilatation différents. De bons résultats ont été obtenus avec un support de molybdène (Fig. 15) puisque des films de dimensions $0,02 \times 5 \times 60 \mathrm{~cm}^{3}$ sont détachés du substrat, qui est, en principe, récupérable pour un nouveau dépôt.

3.1.1.2 Recristallisation du ruban autosupporté. - Le ruban polycristallin subit ensuite une recristal-

a)

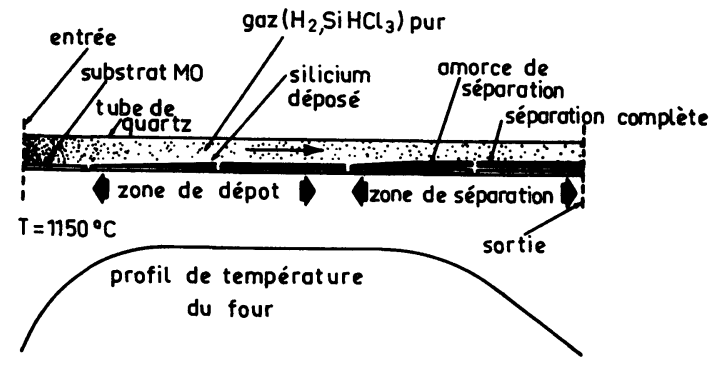

b)

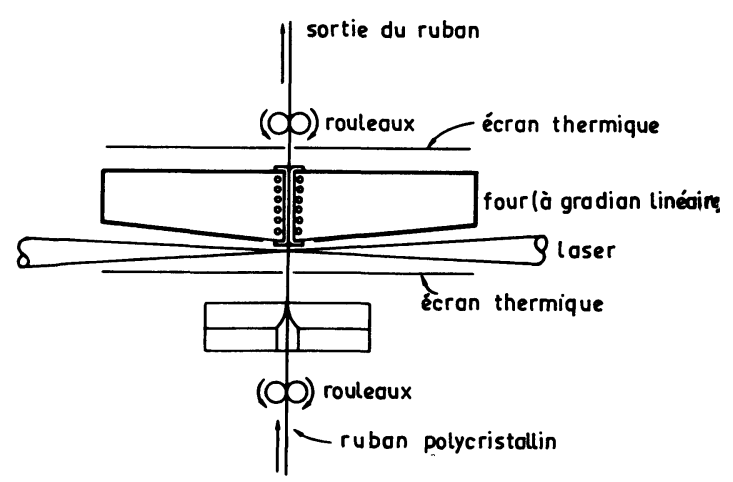

Fig. 15. - $-a$ ) Croissance de silicium (EFG) sur ruban : séparation du film [112, 113] dans un procédé fonctionnant en continu. b) Configuration du four et du faisceau laser utilisés pour la recristallisation des rubans [112].

(a) Polysilicon ribbon formation in a semi-continuous manner. b) Furnace-laser configuration used for regrowth of the films, including heat elements for reducing residual stresses.]
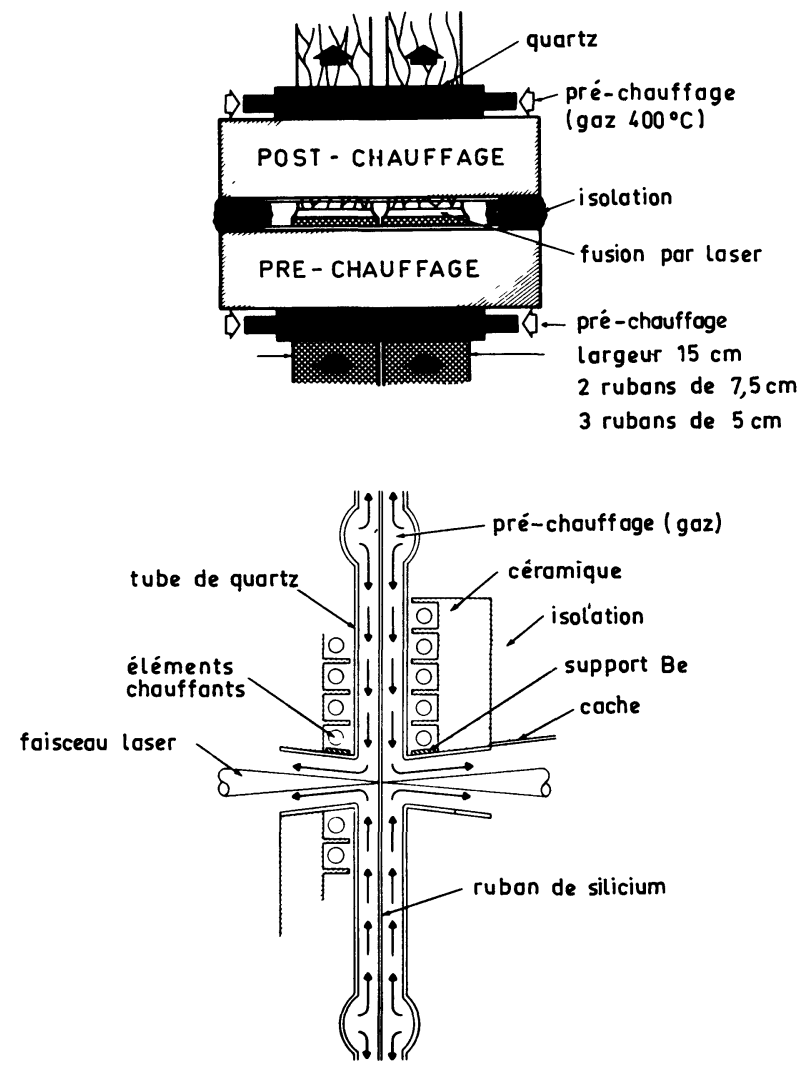

Fig. 16. - a) Principe d'un ensemble de cristallisation laser pour plusieurs rubans [112] ; b) Principe du four destiné à la recristallisation laser.

[a) Principle of a growth apparatus for multiple ribbon; b) Purgeable furnace design in use for laser beam growth.]

lisation par fusion de zone induite par un laser de puissance $\mathrm{CO}_{2}(10,6 \mu \mathrm{m})$, ce qui produit une monocristallisation si un germe orienté initie la croissance (Fig. 16). Les températures atteintes théoriquement avec un laser de puissance $400 \mathrm{~W}$ pour différentes vitesses de croissance du ruban sont données sur la figure 17. Un bon couplage du laser $\mathrm{CO}_{2}$ a nécessité la mise en place d'un four de préchauffage ainsi que d'un réflecteur sphérique pour limiter les pertes de puissances par réflexion sur le silicium fondu.

Pour conserver une zone fondue stable et de faible hauteur, il est nécessaire de maintenir la vitesse de tirage inférieure à $12 \mathrm{~cm} / \mathrm{min}$ pour un film d'épaisseur $100 \mu \mathrm{m}$. La largeur du ruban peut atteindre actuellement $7,5 \mathrm{~cm}$, au-delà des déformations permanentes apparaissent sur les bords, dues à des gradients thermiques non optimalisés.

L'aspect, les densités de dislocations ainsi que les longueurs de diffusion de ce matériau, qui est en fait non entièrement monocristallisé, sont représentés sur la figure 18. Notons que les longueurs de diffusion s'améliorent quelque peu après la réalisation de la cellule par suite du piégeage ou d'une redistribution de certaines impuretés.

Les rendements de conversion des cellules solaires préparées à l'aide de ce matériau sont typiquement de 


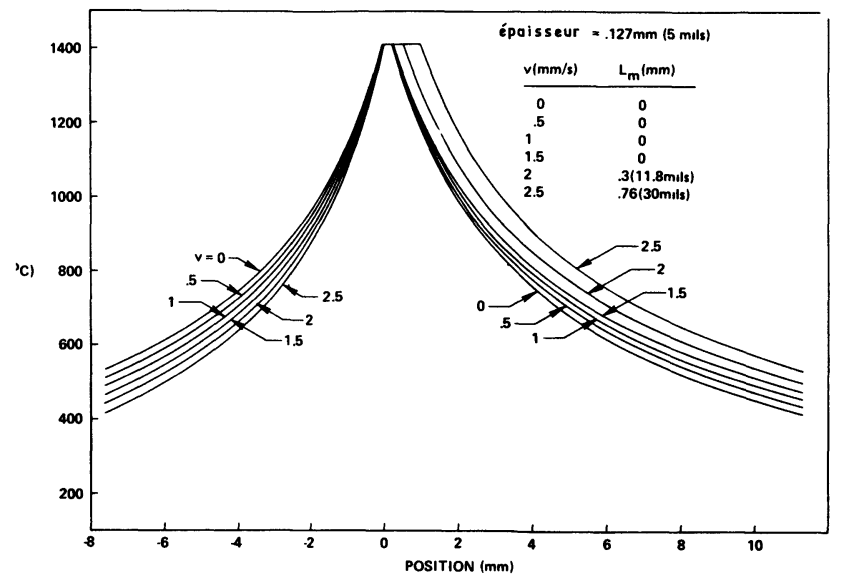

Fig. 17. - Répartition des températures calculées au voisinage de la zone fondue pour différentes vitesses de croissance [112]. $L_{\mathrm{m}}$ représente un éventuel excès de longueur de diffusion dû à une dissipation insuffisante de la chaleur latente.

[Calculated thermal profiles for laser ribbon growth at various velocities. $L_{\mathrm{m}}$ represents an eventual excess semi-molten region length due to an instability to remove latent heat rapidly enough.]

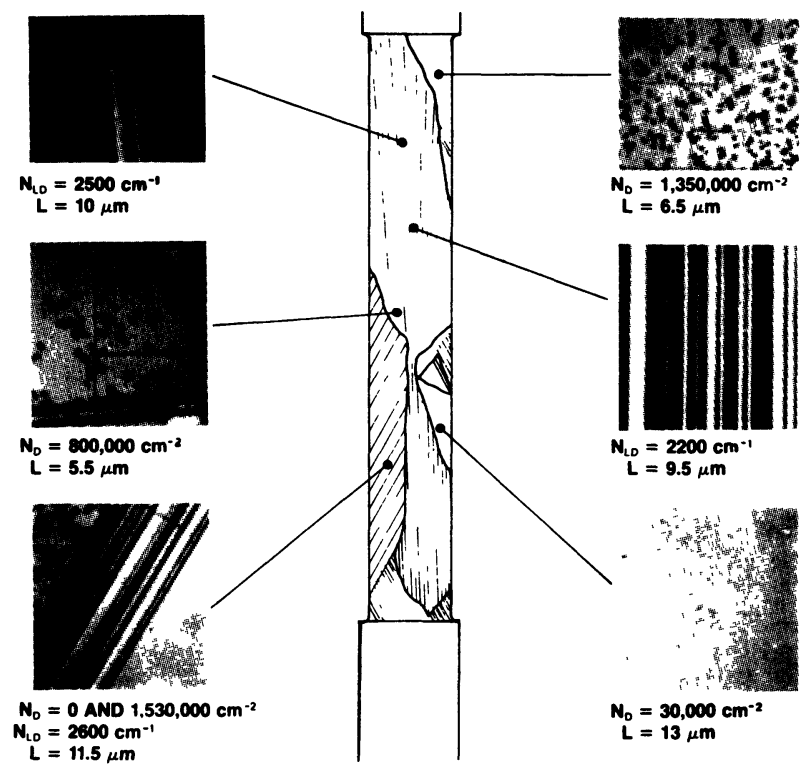

Fig. 18. - Densité de dislocations linéaires $\left(N_{\mathrm{t} . \mathrm{D}}\right)$ et ponctuelles $\left(N_{\mathrm{D}}\right)$ et longueur de diffusion $L$ d'un ruban recristallisé par laser [112].

[Linear $\left(N_{\mathrm{LDD}}\right)$ and point dislocation $\left(N_{\mathrm{D}}\right)$ densities and diffusion length (surface photovoltage technique) on a ribbon sample.]
$8-9 \%$, bien que des valeurs de $12 \%$ aient été mesurées sur certains échantillons.

3.1.2 Recristallisation d'une couche déposée sur un substrat. - Jusqu'ici nous n'avons considéré que l'homo- ou l'hétéro-épitaxie de silicium sur un substrat monocristallin. Mais il est également possible de recristalliser partiellement des films déposés sur un substrat amorphe, malheureusement les épaisseurs sont encore trop faibles $(<1 \mu \mathrm{m})$ pour réaliser des cellules ayant des rendements de conversion suffisants. Nous reportons sur le tableau $\mathrm{V}$ les dimensions des polycristaux dans un film de $5000 \AA ̊$ d'épaisseur dopé par implantation, déposé sur une couche de $\mathrm{Si}_{3} \mathrm{~N}_{4}$. On constate que l'accroissement de la taille des cristaux est bien plus important qu'après recuit thermique conventionnel.

3. 2 RÉALISATION DE LA JONCTION. - La recristallisation induite par laser a été mise en œuvre pour la préparation de la barrière de potentiel de trois manières différentes, à savoir sur des :

3.2.1 Jonctions diffusées conventionnelles. - Rappelons que toutes les cellules solaires commercialisées à l'heure actuelle sont à jonctions diffusées (essentiellement $\mathrm{N}^{+} / \mathrm{P}$ ). La barrière de potentiel est obtenue par diffusion à haute température $\left(850-1100{ }^{\circ} \mathrm{C}\right)$, d'un dopant convenablement choisi, un procédé universellement employé dans la technologie des composants. Malheureusement, tous les dopants introduits ne sont pas électriquement actifs, soit par suite de précipitations au voisinage de la surface, lorsque la solubilité limite est dépassée [120-121], soit par suite de la formation de paires dopant-lacune [122]. Solubilité limite chimique et activité électrique maximale ne coïncident plus au-dessus d'un certain seuil.

3.3 RÉACTIVATION DES DOPANTS. - Il a été montré récemment que la fraction de dopants inactive peut être notablement réduite par une impulsion laser d'énergie $1-1,5 \mathrm{~J} / \mathrm{cm}^{2}[66,123-126]$. En effet, la résistivité de la couche superficielle chute notablement, comme il est décrit dans un autre article de ce numéro [127]. Au moment de la fusion de la couche diffusée, tous les précipités et associations sont dissous. Comme la recristallisation intervient très rapidement, les impuretés littéralement gelées dans le cristal n'auront pas la possibilité de migrer sur des distances notables pour rejoindre les sites de précipitation ou des centres chargés [128]. Il en résulte que les solubilités limites de dopage actif sont notablement accrues [129-131]. Cet

Tableau V. - Recristallisation d'un film de silicium d'épaisseur $5000 \AA$ déposé sur $\mathrm{Si}_{3} \mathrm{~N}_{4}$ et implanté par $5 \times 10^{14}$ ions $\mathrm{B}^{+} / \mathrm{cm}^{2}$.

Vieillissement

Avant recuit Recuit laser

Recuit thermique classique

900-1200 $\left(1000^{\circ} \mathrm{C}\right)$ après laser

$25 \times 2 \mu^{2}$ 


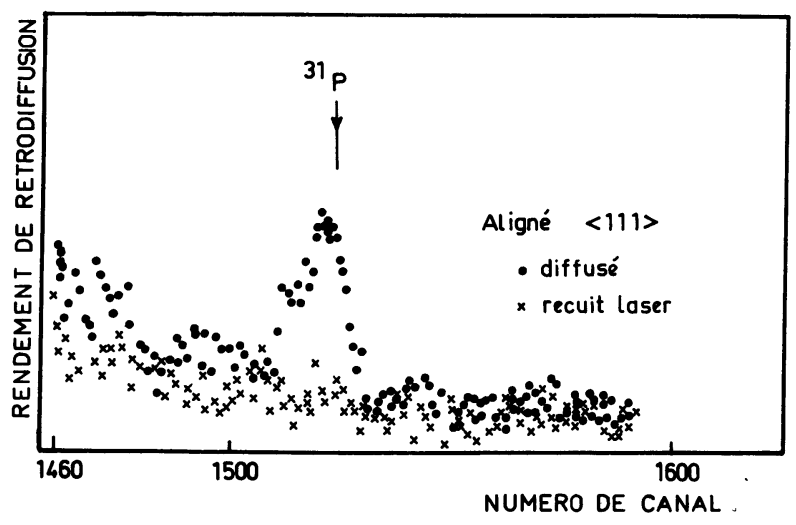

Fig. 19. - Spectre de rétrodiffusion (RBS) en conditions de canalisation suivant l'axe $\langle 111\rangle$ de particules ${ }^{4} \mathrm{He}^{+}$tombant sur du silicium comportant une couche superficielle de phosphore diffusé avant et après recuit laser [126].

[Rutherford backscattering under channelling conditions spectra reached on a phosphorous diffused sample before and after laser annealing.]

effet apparaît clairement sur la figure 19 montrant les spectres de rétrodiffusion d'une jonction diffusée phosphore, observé en conditions de canalisation avant et après recuit laser. La concentration de phosphore interstitiel décroît notablement après recuit.

Par ailleurs, mentionnons qu'une redistribution des dopants se produit également lors du recuit (Fig. 20), ce qui modifie complètement le profil de diffusion initial.

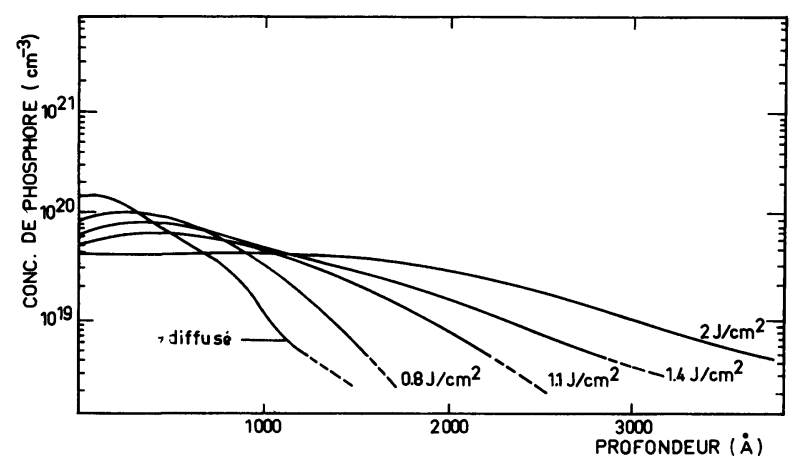

Fig. 20. - Profil de la concentration de phosphore mesuré par SIMS pour différentes énergies d'un laser à rubis [126].

[Phosphorous profile concentration as measured by SIMS after laser annealing of various energy density.]

3.4 EFFETS SUR LES CELLULES. - Un accroissement du dopage de la zone de jonction entraîne une réduction de la résistance série de la cellule et une augmentation de la tension en circuit ouvert $V_{\mathrm{oc}}$. La bonne qualité cristalline après recuit contribue également à un accroissement du courant de court-circuit $I_{\mathrm{SC}}$. Cependant, aux très fortes énergies laser, il y a une augmentation de la profondeur de la jonction entraînant une perte de performances. Ceci a pu être constaté sur une cellule commerciale [126] pour laquelle $V_{\text {oc }}$ a dépassé
$600 \mathrm{mV}$ (Fig. 21) et le rendement de conversion est passé de 11,8 à $12,8 \%$. S'il nous semble que l'application de cette technologie aux cellules conventionnelles sera limitée, des améliorations notables pourront, par contre, être obtenues sur les cellules opérant sous concentration [132].

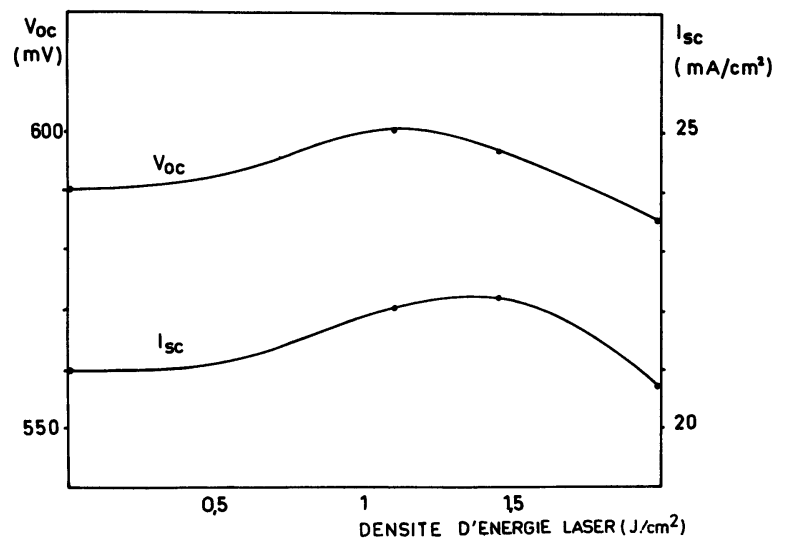

Fig. 21. - Variation de la tension en circuit ouvert $\left(V_{\mathrm{oc}}\right)$ et du courant de court-circuit $\left(I_{\mathrm{SC}}\right)$ en fonction de l'énergie d'un laser rubis pour une cellule diffusée phosphore après recuit laser [126].

[Open circuit voltage $\left(V_{\mathrm{oc}}\right)$ and short circuit current $\left(I_{\mathrm{SC}}\right) v s$. ruby laser energy density for a laser annealed diffused cell.]

3.4.1 Jonctions implantées. - L'implantation ionique est une technique de dopage superficiel qui s'est imposée au cours de ces dernières années dans la technologie des circuits intégrés. En effet, elle offre de nombreux avantages, notamment un contrôle aisé de la concentration et de la pénétration des dopants, un dopage peu profond, très uniforme et sans contamination, même sur des aires importantes. Dès 1967, des cellules solaires ayant des rendements de $11 \%$ (AMO) [133] ont été préparées par cette technique. Le principal inconvénient de cette technologie était jusqu'ici la difficulté de guérir complètement les dommages résultant du bombardement ionique. Le recuit laser offre des possibilités nouvelles qui sont étudiées par la plupart des groupes, tant en vue d'applications aux circuits intégrés qu'aux photopiles.

3.5 Techniques D’implantation. - Essentiellement deux types d'implanteurs sont employés :

- Implanteur conventionnel : les dopants sont ionisés dans une source d'ions, puis extraits du plasma, ạccélérés sous une THT de $10-100 \mathrm{kV}$, avant d'être triés dans un aimant d'analyse, de sorte qu'un faisceau de dopants isotopiquement pur tombe sur l'échantillon. Les intensités de faisceau sont très variables suivant la nature de la source et des dopants : dans les installations conventionnelles elles sont de l'ordre $\mathrm{du} \mu \mathrm{A} / \mathrm{cm}^{2}$, mais des ensembles sophistiqués délivrent des faisceaux de l'ordre du $\mathrm{mA} / \mathrm{cm}^{2}$; malheureusement leur coût est élevé.

Le recuit thermique ne modifie, en général, pas sensiblement la distribution des dopants, toutefois, 
une diffusion accélérée vers la surface (out diffusion) se produit pour certains dopants (Tableau VI). De plus, l'incorporation au réseau est parfois difficile, même à haute température suivant la nature du dopant et l'intensité du faisceau (dose rate effect). En parti-

Tableau VI. - Perte de dopants au cours de recuits thermiques (profondeur d'implantation $300 \AA$ ).

\begin{tabular}{crrrrrr}
$\begin{array}{c}\text { Température } \\
\text { de recuit }\end{array}$ & Ga & In & \multicolumn{1}{c}{ T 1} & As & pb & Bi \\
- & - & - & - & - & - & - \\
$20^{\circ} \mathrm{C}$ & 100 & 100 & 100 & 100 & 100 & 100 \\
$800^{\circ} \mathrm{C}$ & 100 & 65 & 100 & 100 & 100 & 100 \\
$950^{\circ} \mathrm{C}$ & 15 & 10 & 60 & 80 & 90 & 85 \\
$1050^{\circ} \mathrm{C}$ & 5 & 5 & 10 & 30 & 20 & 15 \\
$1100^{\circ} \mathrm{C}$ & 5 & 5 & 5 & 5 & 5 & 5
\end{tabular}

culier, pour les faisceaux les plus intenses, la recristallisation et l'incorporation sont très incomplètes. Sur le tableau VII nous avons rassemblé quelques données pour différents dopants pénétrant de $300 \AA$ dans le silicium.

Le recuit laser produit une redistribution complète des dopants et une incorporation au réseau parfaite (voir ci-dessus et Tableau VIII).

Tableau VIII. - Incorporation des dopants par recuit laser (implantation d'ions bore de $35 \mathrm{keV}$ dans $\mathrm{Si}$ ) [153].

Dose implantée $\left(\mathrm{cm}^{-2}\right)$

Concentration de porteurs après recuit laser $\left(1,7 \mathrm{~J} / \mathrm{cm}^{2}\right)$

Concentration de porteurs après recuit thermique :

$900^{\circ} \mathrm{C} / 30 \mathrm{~min}$

$1100^{\circ} \mathrm{C} / 30 \mathrm{~min}$

Concentration de porteurs après recuit laser suivi d'un recuit thermique à :

$600^{\circ} \mathrm{C} / 30 \mathrm{~min}$

$900^{\circ} \mathrm{C} / 30 \mathrm{~min}$

$$
\begin{array}{ll}
3 \times 10^{15} & 2,5 \times 10^{16} \\
4,5 \times 10^{15} & 3,8 \times 10^{16} \\
& \\
1,0 \times 10^{15} & 4,2 \times 10^{15} \\
2,7 \times 10^{15} & 1,4 \times 10^{16}
\end{array}
$$

$4,5 \times 10^{15} 3,6 \times 10^{10}$ $4,5 \times 10^{15} 1,5 \times 10^{16}$
- Implanteur simplifié [134-135] : dans ce cas, les ions sont produits par décharge dans un gaz contenant le dopant. Après extraction et accélération, ils tombent directement sur l'échantillon, sans aimant de séparation. Ceci conduit à une structure très courte, pour laquelle des faisceaux de $1 \mathrm{~mA} / \mathrm{cm}^{2}$ peuvent être obtenus très économiquement.

Comme indiqué plus haut, la recristallisation par recuit thermique classique est inefficace. Par contre, l'absorption de la lumière d'un laser à rubis est très forte dans la zone amorphisée puisque le coefficient d'absorption est environ dix fois plus important que dans le silicium cristallin. Il en résulte une bonne recristallisation, tandis que le dopant est redistribué (Fig. 22) et qu'une partie du fluor s'échappe.
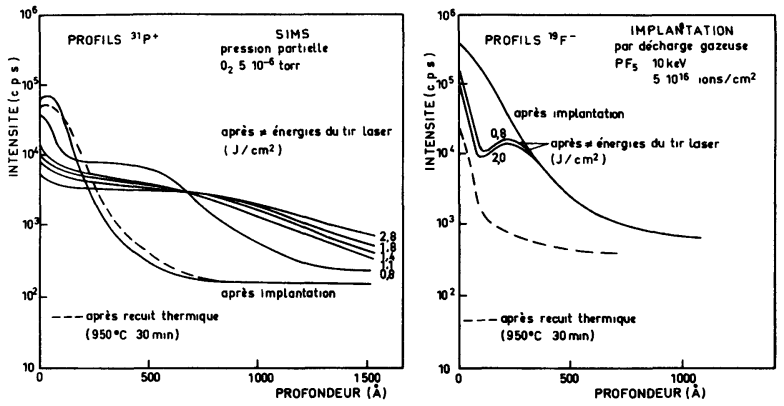

Fig. 22. - Profils de concentration de phosphore et de fluor implantés par effluvage après différents recuits laser. Le profil après un recuit thermique est donné à titre de comparaison [159].

[SIMS phosphorus and fluorine concentration profiles implanted by the glow discharge technique after various laser beam annealing. Thermal annealing effect is given for comparison.]

3.6 Cellules solaires. - Essentiellement trois groupes : Oak Ridge [136], Lockheed [137] et Bologne [138] utilisent l'implantation conventionnelle suivie d'un recuit laser pour préparer des cellules solaires. Les profondeurs de jonctions varient suivant

\begin{tabular}{|c|c|c|c|c|c|c|c|}
\hline \multirow{2}{*}{$\begin{array}{l}\text { Nature } \\
\text { du } \\
\text { dopant }\end{array}$} & \multirow{2}{*}{$\begin{array}{l}\text { Energie } \\
(\mathrm{keV})\end{array}$} & \multirow{2}{*}{$\begin{array}{l}\text { Parcours } \\
\text { théorique } \\
(\AA)\end{array}$} & \multirow{2}{*}{$\begin{array}{c}\text { Largeur } \\
\text { gaussienne } \\
(\AA)\end{array}$} & \multirow{2}{*}{$\begin{array}{c}\text { maximale } \\
\text { pour une dose } \\
\text { de } 10^{14} \mathrm{~cm}^{-2} \\
\left(\mathrm{~cm}^{-3}\right)\end{array}$} & \multirow{2}{*}{$\begin{array}{c}\text { Solubilité } \\
\text { limite } \\
\text { dans silicium } \\
\left(\mathrm{cm}^{-3}\right)\end{array}$} & \multicolumn{2}{|c|}{$\begin{array}{l}\text { Incorporation au réseau }(\%) \\
\text { dose } 10 \mathrm{E} 14 \mathrm{cmE}-3\end{array}$} \\
\hline & & & & & & recuit $600^{\circ} \mathrm{C}$ & recuit $900^{\circ} \mathrm{C}$ \\
\hline - & - & - & - & - & - & - & - \\
\hline B & 7 & 300 & 130 & $3,1 \times 10^{19}$ & $50 \times 10^{19}$ & & $90 \%$ \\
\hline $\mathrm{Al}$ & 20 & 294 & 110 & $3,6 \times 10^{19}$ & $\times 10^{19}$ & & $10 \%$ \\
\hline $\mathrm{Ga}$ & 40 & 280 & 67 & $6,0 \times 10^{19}$ & $\times 10^{19}$ & & $8 \%$ \\
\hline In & 50 & 273 & 47 & $8,5 \times 10^{19}$ & $0,2 \times 10^{19}$ & & $1 \%$ \\
\hline $\mathrm{Tl}$ & 50 & 246 & 28 & $14,3 \times 10^{19}$ & $0,02 \times 10^{19}$ & & \\
\hline $\mathbf{N}$ & 10 & 274 & 132 & $3,0 \times 10^{19}$ & & & \\
\hline $\mathbf{P}$ & 20 & 260 & 94 & $4,2 \times 10^{19}$ & $\times 10^{19}$ & $20 \%$ & $10 \%$ \\
\hline As & 40 & 271 & 62 & $6,4 \times 10^{19}$ & $\times 10^{19}$ & $25 \%$ & $20 \%$ \\
\hline $\mathrm{Sb}$ & 50 & 269 & 45 & $8,9 \times 10^{19}$ & $4,5 \times 10^{19}$ & $25 \%$ & $10 \%$ \\
\hline $\mathrm{Bi}$ & 50 & 245 & 27 & $14,8 \times 10^{19}$ & $0,08 \times 10^{19}$ & $10 \%$ & $1 \%$ \\
\hline
\end{tabular}
la nature et l'énergie du dopant entre 0,3-0,6 $\mu \mathrm{m}$. Les

Tableau VII. - Incorporation des différents dopants implantés après recuits thermiques.

Ions 'de même parcours 


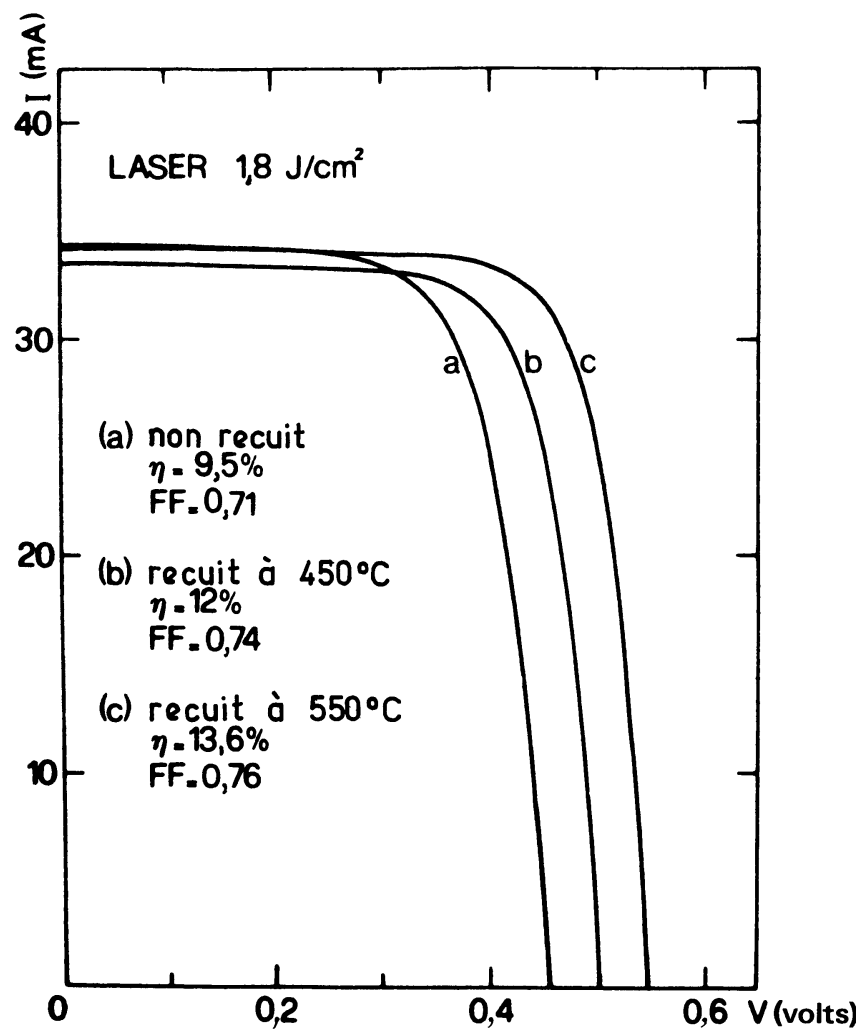

Fig. 23. - Caractéristiques $I-V$ sous éclairement d'une cellule solaire obtenue par implantation $\mathrm{de}^{31} \mathrm{P}^{+}\left(100 \mathrm{keV}, 1 \times 10^{15} \mathrm{~cm}^{-2}\right)$ avec un recuit laser $\left(1,8 \mathrm{~J} / \mathrm{cm}^{2}\right)$ suivi d'un recuit thermique à plusieurs températures [139].

$\left[I-V\right.$ characteristics under illumination of a cell prepared by ${ }^{31} \mathrm{P}^{+}$ implantation $\left(100 \mathrm{keV}, 1 \times 10^{15} \mathrm{~cm}^{-2}\right)$ after laser annealing $(1.8 \mathrm{~J})$ followed by various thermal treatments.]

rendements de conversion publiés varient de 10-15\%, pour des monocristaux, suivant les conditions expérimentales (Fig. 23). Dans la plupart des travaux publiés, celles-ci ne sont pas encore optimalisées.

Très peu d'articles ont été consacrés jusqu'ici [138] à l'effet d'un recuit laser sur la durée de vie des porteurs dans le substrat; des résultats assez contradictoires ont été obtenus.

L'implantation ionique simplifiée (" incrustation d'ions ») étudiée à Strasbourg [134-135] conduit après recristallisation laser à des couches superficielles de résistance 20-30 $\Omega / \square$, inférieure à celle des jonctions diffusées conventionnelles. Les profondeurs de jonctions sont comprises entre 0,15 et $0,20 \mu \mathrm{m}$.

Les rendements de conversion pour les monocristaux sont de l'ordre de $11 \%$ et supérieurs à $9 \%$ [139] pour les films polycristaux (EFG-ribbon) pour des conditions expérimentales non encore optimalisées. Notons que la réalisation de la jonction sur substrat polycristallin par implantation permet d'éviter les risques de précipitation des dopants aux joints de grains, qui se produit généralement au cours des diffusions thermiques conventionnelles. Pour tirer le meilleur profit de cette technique économique, il faut améliorer nos connaissances sur les dommages résul- tant du bombardement ionique très intense, problème commun à celui qui se présente pour la fabrication des circuits électroniques VLSI.

3.6.1 Films déposés en surface. - Il a été montré tout récemment par les groupes d'Oak Ridge [123] et de Strasbourg [140-141] que le recuit laser permettait de former directement des jonctions P-N sans diffusion ni implantation.

3.7 TeCHNIQUe EXPÉRIMENTALE. - On dépose par une méthode quelconque (dépôt sous vide, pulvérisation...) un film d'épaisseur inférieure à $100 \AA$ à la surface du silicium convenablement préparé. Au cours de la fusion induite par un laser pulsé, le dopant est dissous et redistribué dans le silicium liquide. Actuellement, B, Al [123], Sb [140-141], As et Ga [142] ont ainsi été introduits, formant un nouveau type de jonction alliée. Après recristallisation, une bonne qualité cristalline et une très bonne incorporation $\mathrm{du}$ dopant dans le réseau ont pu être mises en évidence. Par exemple pour $\mathrm{Sb}$, les mesures de rétrodiffusion de particules chargées ont montré que pour un dépôt de $10^{16}$ at $/ \mathrm{cm}^{2}$ presque tout l'antimoine était en site substitutionnel (Fig. 24). Un profil de distribution de dopants est reporté sur la figure 25.

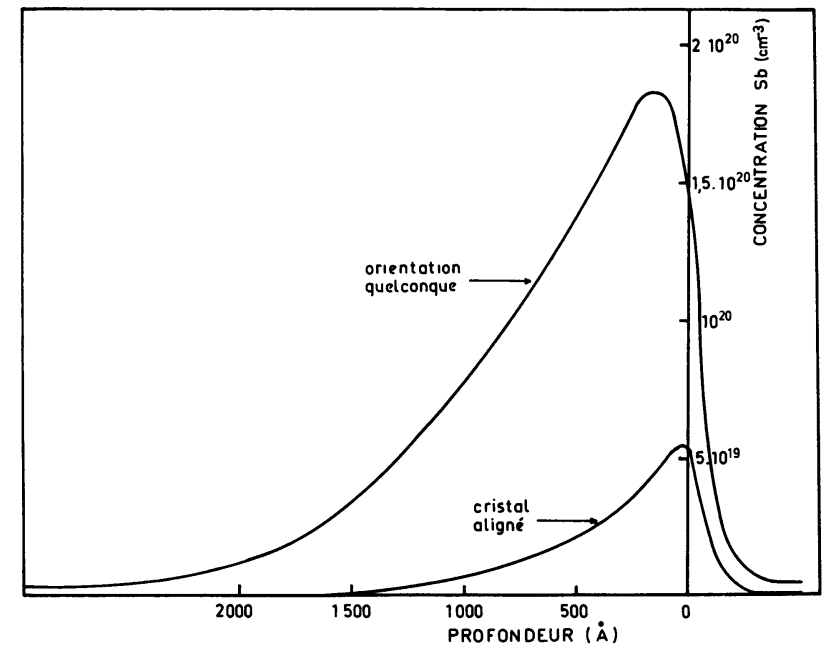

Fig. 24. - Spectres de rétrodiffusion (RBS) de particules ${ }^{4} \mathrm{He}^{+}$ d'une surface de silicium recouverte d'un film d'antimoine après fusion laser [141].

[RBS spectra of antimony deposited on a silicon cristal molten by a laser pulse.]

3.8 Cellules solaires. - Le très fort dopage au voisinage immédiat de la surface conduit à des résistances superficielles très faibles $(20 \Omega / \square)$, ne pouvant être atteintes pour les cellules diffusées classiques. Les premiers résultats font état de rendements de conversion de $10-11 \%$ pour B et $\mathrm{Al}$ [123] et plus de $14 \%$ pour $\mathrm{Sb}$ [141] (Fig. 26).

4. Contacts ohmiques. - Sans entrer dans les détails, mentionnons que des siliciures ou des alliages avec 


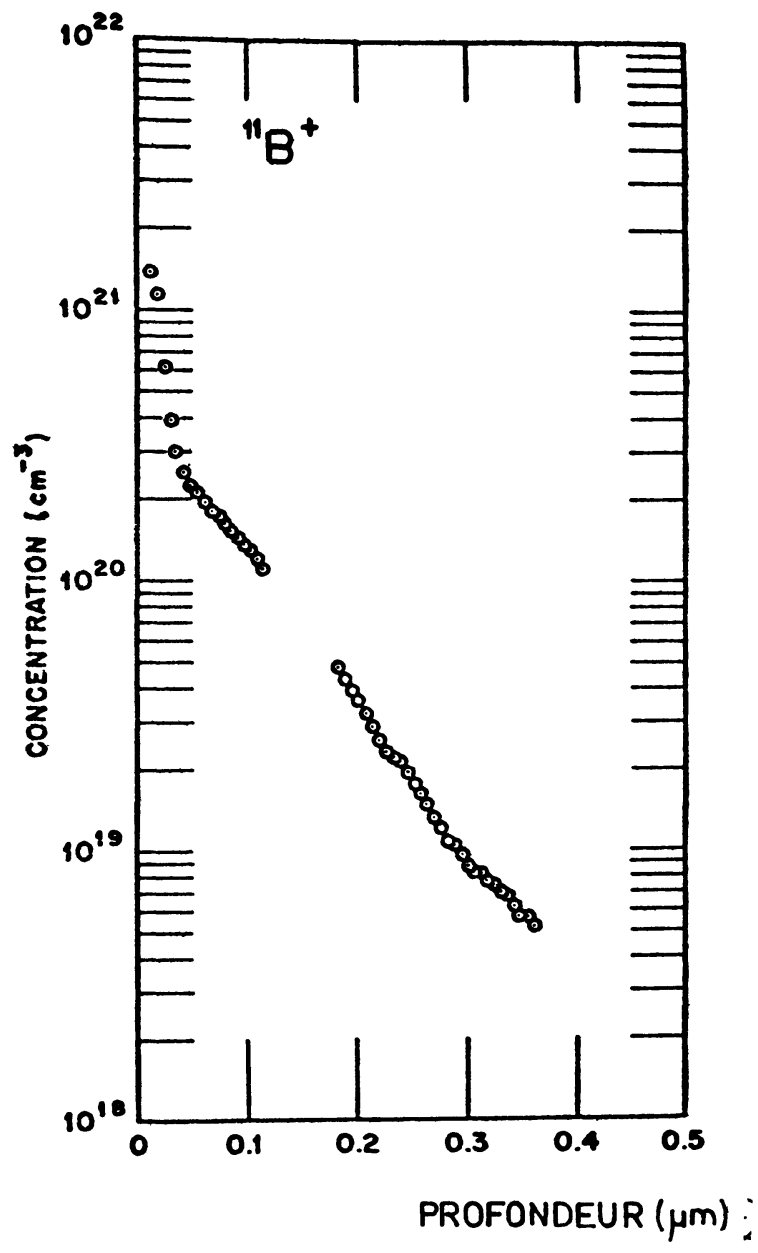

Fig. 25. - Profil de concentration (SIMS) de bore déposé à la surface du silicium et irradié par une impulsion laser [123].

[SIMS concentration profile of boron deposited at the surface of silicon after laser irradiation.]

nombreux éléments ont été obtenus. Citons Pt, Pd, Ni [143], Co, Mo, Pd [144] et la formation de dépôts de $\mathrm{WSi}_{2}, \mathrm{NiSi}_{2}, \mathrm{Pd}_{4} \mathrm{Si}, \mathrm{Pd}_{2} \mathrm{Si}$ [145]. Ces travaux sont encore en pleine évolution à l'heure actuelle, il semble que différents composés peuvent être obtenus suivant l'épaisseur du film déposé, la nature et l'énergie du laser. Certains de ces composés ne sont pas produits au cours de recuits conventionnels.

5. Conclusion. - L'introduction du laser de puissance dans la technologie des semiconducteurs et en particulier dans celle des cellules solaires, constitue un des faits marquants de ces deux dernières années. La préparation de films de silicium à l'aide de cette méthode offre des possibilités intéressantes, mais elle n'est pas immédiatement applicable à une production de masse. Par contre, la préparation de la jonction sans chauffage à température élevée constitue un progrès notable. L'absence de contamination des zones hors de la jonction supprime de nombreux traitements de surface de masquage et de décapage. Dans ces conditions, on peut penser que des lignes de

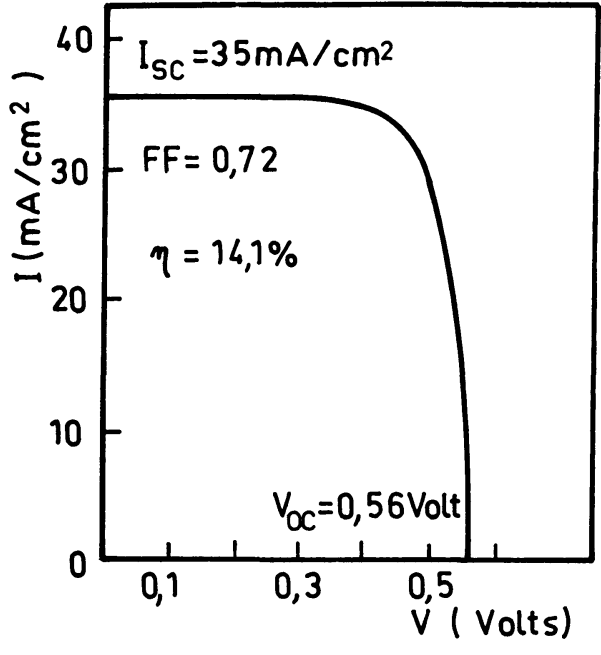

Fig. 26. - Caractéristiques $I-V$ sous éclairement (AM1) d'une cellule obtenue par dépôt d'antimoine à la surface du silicium et irradié par laser [141].

[I-V characteristics under illumination (AM1) of a solar cell prepared by first depositing an antimony layer on silicon and then laser shining.]

production automatisées verront le jour, ce qui devrait entraîner une baisse substantielle des coûts de fabrication.

Parallèlement au développement du recuit laser, le groupe Spire [146-149] a développé le recuit par canon à électrons pulsé. Lequel sera le plus intéressant pour la fabrication des photopiles ? Il est difficile de le dire à l'heure actuelle puisque deux études [150-151] comparatives seulement ont été publiées à ce jour. D'après ces travaux, qui ont porté sur la recristallisation de couches implantées, il semble que :

- La qualité cristalline obtenue après implantation à $100 \mathrm{keV}$ et recuit laser est supérieure à celle produite par recuit par canon à électrons (Tableau IX). Pour des couches implantées à $35 \mathrm{keV}$, les deux recuits sont identiques. Des problèmes d'uniformité du faisceau d'électrons semblent exister.

- Le canon à électrons produit une contamination de la surface par du tungstène arraché au filament.

- Des impulsions successives d'électrons produisent des dommages en surface (" cracks »), alors que jusqu'à 13 impulsions laser ont été mises en œuvre avec le laser rubis sans dommages.

Ces résultats ne sont évidemment pas suffisants pour tirer une conclusion définitive. Aussi avons-nous cherché à regrouper les principales caractéristiques des deux méthodes sur le tableau $X$. La facilité de changer les profondeurs recuites constitue un avantage pour les électrons, par contre, le laser peut fonctionner dans l'atmosphère ambiante, sans recours au vide; les balayages de faisceaux sont plus aisés à obtenir dans ce cas. La fiabilité de chaque technique sera peut-être le facteur déterminant. 
Tableau IX. - Comparaison entre recuits par laser et par canon à électrons.

I. Laser pulsé (rubis) sur couche implantée $\mathrm{As}^{+} 100 \mathrm{keV}$, dose $1,4 \times 10^{16} \mathrm{~cm}^{-2}, 1,1 \times 10^{16} \mathrm{~cm}^{-2}$.

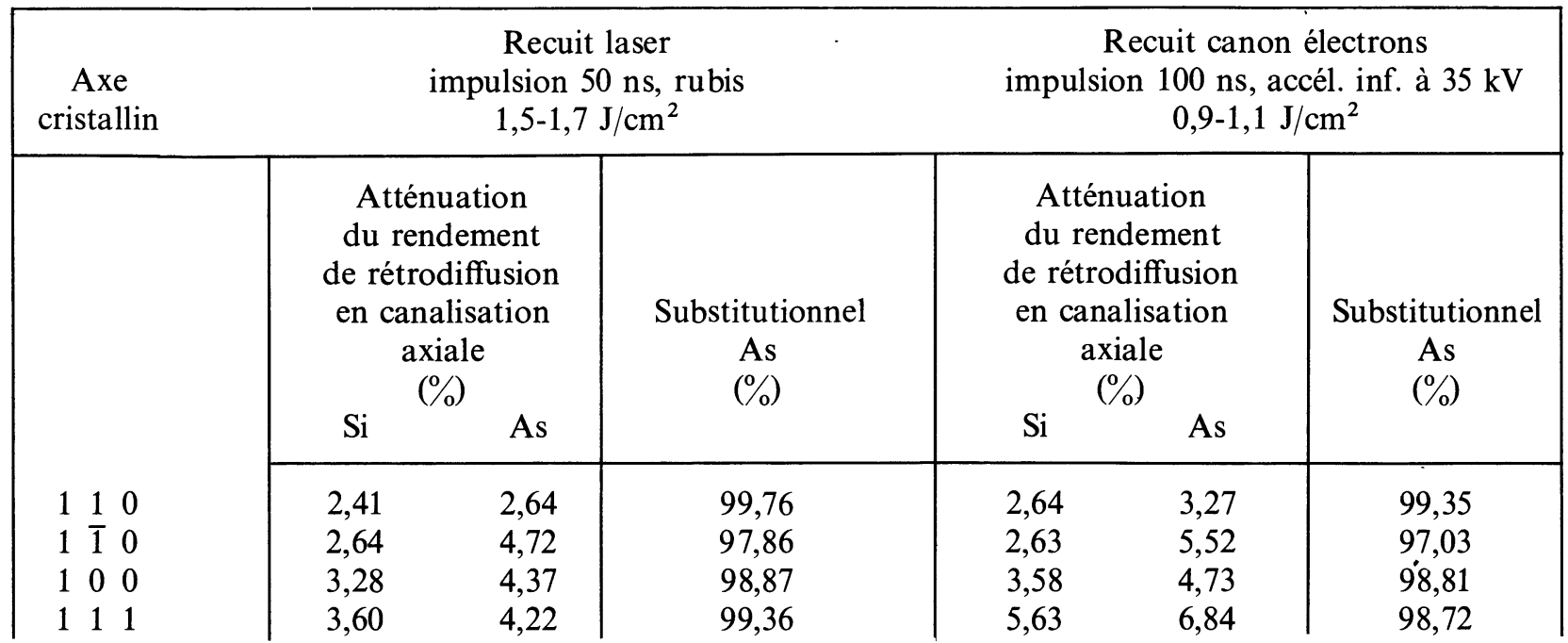

II. Laser continu argon balayé sur une couche implantée $\mathrm{As}^{+} 100 \mathrm{keV}$, dose $10^{16} \mathrm{~cm}^{-2}$.

\begin{tabular}{|l|c|c|}
\hline & $\begin{array}{c}\text { Recuit laser } \\
\mathrm{CW} \text { argon, balayé } \\
15 \mathrm{~W}\end{array}$ & $\begin{array}{c}\text { Recuit canon à électrons } \\
\text { impulsion 100 ns, accél. 31 } \mathrm{kV} \\
14 \mathrm{~W}\end{array}$ \\
\hline Température du substrat & $350^{\circ} \mathrm{C}$ & $50^{\circ} \mathrm{C}$ \\
Puissance absorbée $(\mathrm{W} / \mu)$ & 0,17 & 0,14 \\
Vitesse de balayage $(\mathrm{cm} / \mathrm{s})$ & 12,5 & 2,5 \\
Environnement & ambiant & vide $\left(10^{-4}\right.$ torr $)$ \\
Atténuation en rétrodiffusion en conditions de & 11 & 11 \\
canalisation $(\%)$ & 22 & 21 \\
As ${ }^{+}$non substitutionnel, se trouvant dans l'axe & 20 & 28 \\
$100(\%)$ & $1,13 \times 10^{21}$ & $1,07 \times 10^{21}$ \\
$\mathrm{As}^{+}$inactif $(\%)$ & &
\end{tabular}

Tableau X. - Lasers et canons à électrons pour recuit superficiel pulsé.

\begin{tabular}{|c|c|c|}
\hline & Canon à électrons [146-149] & Laser pulsé \\
\hline Energie disponible & $1 \mathrm{~J} / \mathrm{cm}^{2}$ & $1-10 \mathrm{~J} / \mathrm{cm}^{2}$ \\
\hline Durée d'une impulsion (largeur & & \\
\hline à mi-hauteur, mi-puissance) & $10-200 \mathrm{~ns}$ & $10-200 \mathrm{~ns}$ \\
\hline Diamètre faisceau & $75 \mathrm{~mm}$ & $25 \mathrm{~mm}$ \\
\hline Balayage faisceau & Difficile, surface endommagée & aisé \\
\hline $\begin{array}{l}\text { Taux de production horaire } \\
\text { (nombres de plaquettes; } \\
\text { pour mémoire, diffusion ther- }\end{array}$ & & \\
\hline mique $=100)$ & Supérieur à 60 & 110 \\
\hline Atmosphère ambiante & Vide, au moins $10^{-4}$ torr & Atmosphère ambiante \\
\hline Profondeur de pénétration & Aisément variable en agissant sur THT & Fixé par les propriétés optiques \\
\hline $\begin{array}{l}\text { Sensibilité aux propriétés cris- } \\
\text { tallographiques ou de surface } \\
\text { (réflection) }\end{array}$ & & Oui très forte \\
\hline Uniformité du faisceau & Mal connue actuellement & Gaussienne \\
\hline Sensibilité conductivité & Difficile sur isolants & Non \\
\hline
\end{tabular}




\section{Bibliographie}

[1] Shtyrkov, E. I., Khaibullin, I. B., Zaripov, M. M., Galytudinov, M. F. and Bayazitov, R., Sov. Phys. Semicond. 9 (1976) 1309.

[2] Kutukova, O. G. and Strelitson, L. N., Sov. Phys. Semicond. 10 (1976) 265.

[3] Antonenko, A. K., Gerasimenko, N. N., DvurechenSkiI, A. V., Smirnov, L. S. and Tsertlin, G. M., Sov. Phys. Semicond. 10 (1976) 81.

[4] Kachurin, G. A., Nidaev, E. V., Khodyachikhi, A. V. and Kovaleva, L. A., Sov. Phys. Semicond. 10 (1976) 1128.

[5] Bogatyrev, V. A., Gavrilov, A. A., Kachurin, G. A. and Smirnov, L. S., Sov. Phys. Semicond. 10 (1976) 826.

[6] achurin, G. A., Pridachin, N. B. and Smirnov, L. S., Sov. Phys. Semicond. 9 (1976) 946.

[7] Kachurin, G. A., Bogatyrev, V. A., Romanov, S. I., Ion implantation in Semiconductors, Ed. F. Chernow, J. A. Borders, D. Brice (1976) 445.

[8] Bolotov, V. V., Pridachin, N. B. and Smirnov, L. S., Sov. Phys. Semicond. 10 (1976) 338.

[9] Bogatyrev, V. V. and Kachurin, G. A., Sov. Phys. Semicond. 11 (1977) 56.

[10] Khaibullin, I. B., Shtykov, E. I., Zaripov, M. M., Galyautdinov, M. F. and Zarirov, G. G., Sov. Phys. Semicond. 11 (1977) 190.

[11] Kachurin, G. A. and Nideav, E. V., Sov. Phys. Semicond. 11 (1977) 350

[12] Workshop on laser effects in ion implantation semiconductors, Catania (Italy) (1978) Ed. Rimini.

[13] International Conf. on Ion Beam Modification of Materials, Budapest (1978) Hungary.

[14] AIP Proceedings $\mathrm{n}^{\circ} 50$ on laser solid interaction and laser processing materials research society meeting, Boston, U.S.A. (1978) ed. by S. D. Ferris, M. J. Leamy and J. M. Poate.

[15] Electronic Materials Conference, Boulder, U.S.A. (1979).

[16] Laser and Electron Beam Processing of Electronic Materials, Los Angeles, U.S.A. (1979).

[17] Laser and Electron Beam Processing of Materials, Materials Research Society Symposium, Cambridge, U.S.A. (1978).

[18] 14th IEEE Photovoltaic Specialists Conference, San Diego. U.S.A. (1980).

[19] Gold, R. B., Powell, R. A., Gibbons, J. F., Ref. [14].

[20] Tandow, J. L., Nicolet, M. A., Tseng, W. F., Eisen, F. H., Campisano, S. U., Foti, G. and Rimini, E., Appl. Phys. Lett. 34 (1079) 598.

[21] Margalit, S., Fekete, D., Pepper, D. M., Lee, C. P., Yariv, A., Appl. Phys. Lett. 33 (1978) 246.

[22] Kaganov, M. J., Lifshitz, I. M. and Tanotorov, V., Sov. Phys. JETP 4 (1957) 173.

[23] Grinberg, A. A., Mekhtiev, R. F., Ryvkin, S. M., Salmanov, V. M., Yarosmetskiı, I. D., Sov. Phys. Solid State 9 (1967) 1085

[24] Kazkaz, A. G., Thèse, Univ. Scientifique et Technique, Grenoble (1973) France.

[25] Baeri, P., Campisano, S. U., Foti, G., Rimini, E., J. Appl. Phys. 50 (1979) 790

[26] White, C. W., Christie, W. H., Eby, R. E., Wang, J. C., Young, R. T., Clark, G. J., Wood, R. F., J. Electrochem. Soc.

[27] Khaibullin, I. B., Shtyrkov, E. I., Zaripov, M. M., Bajozitov, R. M., Aganov, R. V., Ref. [13] 735.

[28] Khaibullin, I. B., Shtyrkov, E. I., Zaripov, M. M., Bayazitov, R. H. and Galjautdinov, M. F., Radiat. Eff. 36 (1978) 225.

[29] Hfinig, K. H., Klabes, R. and Woittennek. H.. Ref. [13] 707.

[30] Bell, R. O., Toulemonde, M. and Sil Hek1, P., Appl. Phys. 19 (1979) 313.
[31] Muller, J. C., Scharager, C., Toulemonde, M. et Siffert, P., Conférence de la Société Française de Physique, Toulouse (1979) France.

[32] Surko, C. M., Simons, A. L., Auston, D. H., Golovchenko, J. A., Slusher, R. E. and Venkatesan, T. N. C., Ref. [14] 155.

[33] Liau, Z. L., Tsaur, B. Y. and Mayer, J. W., Ref. [12] 147.

[34] LaU, S. S., Mayer, J. W. and Tseng, W. F., Ref. [14] 84.

[35] Lax, M., J. Appl. Phys. 48 (1977) 3919.

[36] LaX, M., Ref. [14] 149.

[37] Morhange, J. F., Kanellis, G., Balkanski, M., Peray, J. F., ICOLE, J. and Croset, M., Ref. [14].

[38] Nakamura, K., Kanoshida, M., Vehara, A. and Tatsumi, R., Ref. [14] 434.

[39] Porter, W. A., Parker, D. L., Richardson, T. W., Ref. [14] 453.

[40] ChU, W. K., MAYER, J. W., Nicolet, M. A., Backscattering Spectroscopy (Academic Press) 1978

[41] Krynicki, J., SUSKI, J., UgniewSKI, J., Grötzschel, R., Klabes, R., Kreissig, U. and Rüdiger, G., Phys. Lett. 61A (1977) 181.

[42] Mustafin, T. N., Kachurin, G. A., Popov, V. P., Pridachin, N. V., Seriapin, V. G., Smirnov, L. S., Ref. [13] 727.

[43] Fot1, G., Della Mla, G., Lethere al Nuove (imento 21 (1978) 89.

[44] Young, R. T., White, C. W., Clark, G. J., Narayan, J., Christie, W. H., Murakami, M., King, P. W. and Kramer, S. D., Appl. Phys. Lett. 32 (1978) 139.

[45] White, C. W., Pronko, P. P., Appleton, B. R., Wilson, S. R. and Narayan, J., Ref. [13] 809.

[46] Foti, G., Campisano, S. U., Rimini, E. and Vitali, G.. J. Appl. Phys. 49 (1978) 2569.

[47] Foti, G., Rimini, E. and Campisano, S. U., Phys. Statu. Solidi (a) 47 (1978) 533.

[48] Foti, G., Rimini, E., Tseng, W. S. and Mayer, J. W., Appl. Phys. 15 (1978) 169.

[49] Baeri, D., Campisano, S. U., Foti, G. and Rimini, E.. Appl. Phys. Lett. 33 (1978) 137.

[50] Celler, G. K., Poate, J. M. and Kimerling, L. C., Appl. Phys. Lett. 32 (1978) 464.

[51] Muller, J. C., Grob, A., Grob, J. J., Stuck, R. and SifFERT, P., Appl. Phys. Lett. 33 (1978) 287.

[52] Muller, J. C., Grob, J. J. and A., Stuck, R. and Siffert, P. Ref. [13] 761.

[53] Hoonhout, D., KerkdiJk, C. B. and Saris, F. W., Phys. Lett. 66 A (1978) 145.

[54] Revesz, P., Farkas, G., Mezey, G. and Gyulai, J., Appl. Phys. Lett. 33 (1978) 431.

[55] Lau, S. S., Tseng, W. F., Nicolet, M. A., Mayer, J. W., ECKardt, R. C. and Wagner, R. J., Appl. Phys. Lett. 33 (1978) 130.

[56] Bean, J. C., Leamy, M. J., Poate, J. M., Rozgonyi, G. A., Sheng, T. T., Williams, J. S. and Celler, G. K., Appl. Phys. Lett. 33 (1978) 227.

[57] Bean, J. C., Leamy, H. J., Poate, J. M., Rozgonyi, G. A., van DeR Ziel, J. P., Williams, J. S. and Celler, G. K., J. Appl. Phys. $\mathbf{5 0}$.

[58] Tamminga, Y., Hoonhout, D., Saris, F. W., Hofker, W. F., Ref. [13].

[59] Tamminga, Y., Eggermont, G. E. S., Hofker, W. K., Hoonhout, D., Garrett, R. and Saris, F. W., Phys. Lett. 69A (1979) 436.

[60] Romanov, S. I., Kachurin, G. A., Smirnov, L. S., KaIbullin, I. B., Shtyrkov, E. I., Bayazitov, R. M., Ref. [13] 777.

[61] Poall. J. M.. Brom . W. L... Li iwi. M. J.. Rodgilks. J. W. Rousseau, D., Rozgonyi. G. A.. Shelnutt, J., Sheng, T. T., Williams, J. S. and Celler, G. K., Ref. [13] 787. 
[62] Narayan, J., Young, R. T. and White, C. W., Ref. [13].

[63] Larson, B. C., White, C. W. and Appleton, B. R., Appl. Phys. Lett. 32 (1278) 801.

[64] Battaglin, G., Della-Mea, G., Drigo, A. V., Bentini. G. G., Servidori, M. and Foti, G., Ref. [13].

[65] Foti, G., Ref. [12] 79.

[66] Narayan, J., J. Appl. Phys. 34 (1978) 312.

[67] Shashkov, Yu. M., Gurevich, V. M., Russ. J. Phys. Chem. 42 (1968) 1082.

[68] Liau, Z. L., Tsaur, B. Y., LaU, S. S., Golecki, I. and Mayer. J. W., Ref. [14] 105.

[69] Auston, D. H., Golowchenko, J. A., Smith, P. R., Surko, C. M. and Venkatesan, T. N. C., Appl. Phys. Lett. 33 (1978) 539.

[70] Williams, J. S., Brown, W. L., Leamy, M. J., Poate, J. M., Rodgers, J. W., Rousseau, D., Rozgonyi, G. A., ShelNutt, J. A. and Sheng, T. T., Appl. Phys. Lett. 33 (1978) 543.

[71] Gat, A., Gibbons, J. F., Magee, T. J., Peng, J., Williams, P., Deline, V. and Evans, C. A., Appl. Phys. Lett. 33 (1978) 389.

[72] Gat, A., Gertberg, L., Gibbons, J. F., Lietoila, A., TilLeR, W., Magee, T. J., Peng, J., Deline, V., Williams, P. and Evans, C. A., Ref. [13] 681.

[73] Gibbons, J. F., Gat, A., Bertberg, L., Lietolla, A., Regolini, J. L., Signon, T. W., Pease, R. F. W., Magee, T. J., Peng, J.. Hong, J., Ref. [14] 365.

[74] Dvurechensky, A. V., Kachurin, G. A. and Aniontnho. A. Kh., Radiat. Eff. 37 (1978) 179.

[75] Smirnov, L. S. and Kachurin, G. A., Ref. [13] 653.

[76] Lysenko, K. S., Nazorov, A. N., Lokshin, M. M. and Kaschieva, S. B., Soviet Phys. Semicond. 11 (1977) 1327.

[77] Mooney, P. M., Young, R. T., Karins, J., Lee, Y. H. and Corbett, J. W., Phys. Status Solidi 48a (1978) K 31.

[78] Benton, J. L., Kimerling, L. C., Miller, G. L., RobinSON, D. A. H., CEller, G. K., Ref. [14] 543.

[79] Johnson, N. M., Gold, P. B., Lietolla, A. and Gibbons, J. F., Ref. [14] 550 .

[80] Johnson, N. M., Gold, R. B. and Gibbons, J. F., Appl. Phys. Lett. 34 (1979) 704.

[81] Young, R. T., White, C. W., Narayan, J., Pronko, P. P., Appleton, B. R., Wilson, S. R. and Mooney, P. M., Ref. [13] 831.

[82] Goltzene, A., Muller, J. C., Schwab, S. et Siffert, P., Conf. de la Société Française de Physique Toulouse (1978) France

[83] Gat, A., Gibbons, J. F., Magee, T. J., Peng, J., Deline, V. R., Williams, P. and Evans, C. A., J. Appl. Phys. 32 (1978) 276.

[84] Auston, D. H., Golovchenko, J. A., Smith, P. R., Surko, C. M. and Venkatesan, T. N. C., Appl. Phys. Lett. 33 (1978) 539.

[85] Celler, G. K., Borutta, R., Brown, W. L., Poate, J. M., Rozgonyi, G. A., Sheng, T. T., Ref. [14] 381.

[86] Dan Forth, S. C., Flint, J. H., Cannon, W. R. and HaGGERTY, J. S., Ref. [14] 659.

[87] Celler, G. K., Ref. [12] 13.

[88] Friedrich Schiller Universität Jena (GRD).

[89] Kazan Physical Technical Institute Academy of Science (USSR).

[90] Novosibirsk, Institut of semiconductor Physics Siberian Branch Academy of Sciences (USSR).

[91] Zentralinstitut für Kernforschung, Rossendorf (GDR).

[92] Institute di Structtura della Materia Universita di Catania (Italy).

[93] Laboratorio Lamel, C.N.R. Bologne (Italy).

[94] FOM Institute for Atomic and Molecular Physics, Amsterdam (Netherlands).

[95] Harvard University Cambridge (Mass), U.S.A.

[96] Bell laboratories Murray Hill, New Jersey (USA).

[97] Oak Ridge National Laboratory, Tennessee (USA).

[98] Institut für angewandte Physik Universität Bern (Switzerland).
[99] Central Research Lab. Hitachi, Tokyo, Faculty of Engineering Science, Osaka (Japan).

[100] Groupe de Physique et Applications des Semiconducteurs CRN Strasbourg (France).

[101] Liu, P. L., Yen, R. and Bloembergen, N., Appl. Phys. Lett. 34 (1979) 864

[102] Kachurin, G. A. and Nidaev, E. V., Sov. Phys. Semicond. 11 (1977) 1178.

[103] Regolini, J. L., Sigmon, T. W., Magee, T. J. and Peng, J., Ref. [14] 393.

[104] Vitali, G., Bertolotti, M. and Foti, G., Appl. Phys. Lett. 33 (1978) 1018.

[105] Vitali, G., Bertolotti, M. and Stagni, L., Ref. [14] 111. [106] Laser Focus, Mai (1979) 30.

[107] Surko, C. M., Simons, A. L., Auston, D. H., GolovchenKo, J. A., Slusher, R. E. and Venkatesan, T. N. C., Appl. Phys. Lett. 34 (1979) 635.

[108] Auston, D. H., Golovchenko, J. A., Simons, A. L. and Surko, C. M., Appl. Phys. Lett. 34 (1979) 777.

[109] Murakami, K., Kawabe, M., Gamo, K., Namba, S. and Aoyagi, Y., Phys. Lett. 70A (1979) 332.

[110] Muralami, K., Gamo, K., Namba, S., Kawabe, M., Aoyagi, Y. and AKASAKA, Y., Ref. [14] 61.

[111] Lesk, I. A., Bagdadi, A., Gurtler, R. W., Ellis, R. J., Wise, J. A., Goleman, M. G., 12th IEEE Photovoltaic Specialists Conference, Baton Rouge (USA) (1976) 173.

[112] Gurtler, R. W., Bagdadi, A., Legge, R. N. and Ellis. R. J., European Photovoltaic Solar Energy Conf. Berlin (W. Germany) (1979) 145, Ed. Reidel.

[113] Gurtler, R. W., Baghdadi, A., Ellis, R. J., Lesk, I. A., J. Electron. Mater. 7 (1978) 441

[114] Chu, T. L. and Singh, K. N., Solid State Electron. 19 (1976) 837.

[115] Chu, T. L., Mollenkoff, H. C., Chu, S. S., J. Electrochem. Soc. 122 (1975) 1681.

[116] Hall, R. N., Gordon Conference on Crystal Growth, New Hampshire (1976) (USA).

[117] CHU, T. L., IEEE Trans. Electron Device 24 (1977) 4.

[118] Schins, W. Jh., Bezemer, J., Daey-Ouwens, L. and RadeLAAR, S., European Photovoltaic Solar Energy Conf., Luxembourg (1977) 143, Ed. Reidel.

[119] Graef, M. W. M., Giling, L. J. and Bloem, J., Ref. [118] 136 .

[120] Schmidt, P. F. and Stickler, R., J. Electrochem. Soc. 111 (1964) 1188

[121] Trumbore, F. A., Bell Syst. Techn. J. 39 (1960) 205.

[122] FAIR, R. B. and TsaI, J. C. C., J. Electrochem. Soc. 124 (1977) 1109.

[123] Narayan, J., Young, R. T., Wood, R. F. and Christie, W. H., Appl. Phys. Lett. 33 (1978) 338.

[124] Cembali, F., Galloni, R., Lolli, R., Pedulli, L. and ZignaNi, F., Ref. [13] 859.

[125] Young, R. T. and Narayan, J., Appl. Phys. Lett. 33 (1978) 14.

[126] Fogarassy, E., Stuck, R., Muller, J. C., Grob, A. and J. J. and SifFERT, P., Ref. [112] 768.

[127] Salles, D., Fogarassy, E., Stuck, R. and Siffert, P., Rev. Phys. Appl.

[128] Campisano, S. U., Ref. [12] 139.

[129] JACKSON, K. A. and Leamy, H. J., Ref. [14] 102.

[130] White, C. W., Narayan, J. and Young, R. T., Ref. [14] 275.

[131] Stuck, R., Fogarassy, E., Muller, J. C., Grob, A. and J. J. and SifFERT, P., Ref. [16].

[132] Fogarassy, E., Stuck, R., Muller, J. C., Salles, D., Siffert, P. et SAlles, Y., Brevet Français 7916968 (1979).

[133] Burril, J. F., King, W. J., Harrisson, S. and McNally, P., IEEE Trans. Electron. Devices 14 (1967) 10.

[134] Muller, J. C., Ponpon, J. P., Grob, J. J. and A., Stuck, R. and SiFfert, P., Ref. [118] p. 897.

[135] Muller, J. C., Grob, A. and J. J., Stuck, R. and Siffert, P. 13th IEEE Photovoltaic Specialists Conf., Washington (USA) (1978) 711 
[136] Young, R. T., White, C. W., Clark, G. L., Narayan, J., Westbrook, R. D. and Christie, W. H., Ref. [118] 861.

[137] Lockheed, voir Laser Focus, Mai (1979) 30.

[138] Zignani, F., Galloni, R., Pedulli, L., Bentini, G. G., Servidori, M., Cembali, F. and Desalvo, A., Ref. [112] p. 213.

[139] Bell, R. O., Ho, K. T., Ravi, K. V., Wald, F. V., Muller, J. C. and SifFert, P., Ref. [112] p. 153.

[140] Bell, R. O., Muller, J. C., Toulemonde, M., Stuck, R. and SifFERT, P., Ref. [14] 299.

[141] Stuck, R., Fogarassy, E., Grob, A., Grob, J. J., Muller, J. C. and SifFerT, P., Ref. [16].

[142] Berger, R., Rudolf, F., Jaccard, L., Roulet, M. E., LÜThy, W., Siregar, M. R. T. and Weber, M. P., IV Int. Conf. on Ion Beam Analysis, Aahrus (1979) (Denmark).

[143] Poate, J. M., Leamy, H. J., Sheng, T. T. and Celler, G. K., Ref. [14] 527.

[144] Van Gurp, G. J., EgGermoni, G. E. J., Tanninga, Y., Tstacy, W. and Gijsbers, J. R. M., Appl. Phys. 35(3) (1979) 273.

[145] Tan, T. Y., Tsu, R., Ho, P. S. and Tu, K. N., Ref. [14] 533.

[146] Greenwald, A. C., Kirkpatrick, A. R., Little, R. C. and MinnucCI, J. A., J. Appl. Phys. 50 (1979) 783.

[147] KirkPatrick, A. R., MinnuCCI, J. A. and Greenwald, A. C., IEEE Trans. Electron. Devices 24 (1977) 429.
[148] Kirkpatrick, A. R., Greenwald, A. C., Minnucci, J. A. and LitTLE, R. G., Ref. [14] 475.

[149] Lay, S. S., Tseng, W. F., Nicolet, M. A., Mayer, J. W., Minnucci, J. A. and Kirkpatrick, A. R., Appl. Phys. Lett. 33 (1978) 235.

[150] Wilson, S. R., Appleton, B. R., White, C. W., NaraYAN, J., Ref. [14] 481.

[151] Regolini, J. L., Sigmon, T. W. and Gibbons, J. F., Appl. Phys. Lett. 35 (1979) 114.

[152] Young, R: T., White, C. W., Narayan, J., Westbrook, R. D., Wood, R. F. and Christie, W. H., Ref. [135] 717.

[153] Narayan, J., Young, R. T. and White, C. W., J. Appl. Phys. 49 (1978) 3912.

[154] White, C. W., Christie, W. H., Appleton, B. R., Wilson, S. R., Pronko, P. P., Magee, C. W., Appl. Phys. Lett. 33 (1978) 662.

[155] White, C. W., Narayan, J. and Young, R. T., Ref. [14] p. 275.

[156] Lullis, A. G., Poate, J. M. and Celler, G. K., Ref. [14] 311.

[157] Williams, J. S., Brown, W. L., Leamy, H. J., Poate, J. M., Rodgers, J. W., Rousseau, D., Rozgonyi, G. A., Shelnutt, J. A. and Sheng, T. T., Ref. [12] p. 98.

[158] Gat, A. and Gibbons, J. F., Appl. Phys. Lett. 32 (1978) 142.

[159] Muller, J. C., Siffert, P., Michel, J. and Fabre, E.. Ref. [17].

[160] Mayer, J. W. and Clogston, P. T., Ref. [12] 1. 